\title{
Stability of boundary element methods for the two dimensional wave equation in time domain revisited
}

\section{$\operatorname{AUTHOR}(S)$ :}

Fukuhara, Mio; Misawa, Ryota; Niino, Kazuki; Nishimura, Naoshi

\section{CITATION:}

Fukuhara, Mio ...[et al]. Stability of boundary element methods for the two dimensional wave equation in time domain revisited. Engineering Analysis with Boundary Elements 2019, 108: 321-338

\section{ISSUE DATE:}

2019-11

URL:

http://hdl.handle.net/2433/244714

\section{RIGHT:}

(c) 2019. This manuscript version is made available under the CC-BY-NC-ND 4.0 license

http://creativecommons.org/licenses/by-nc-nd/4.0/:; The full-text file will be made open to the public on 1 November 2021 in accordance with publisher's 'Terms and Conditions for Self-Archiving'.; この論文は出版社版でありません。引 用の際には出版社版をご確認じ利用ください。; This is not the published version. Please cite only the published version. 


\title{
Stability of boundary element methods for the two dimensional wave equation in time domain revisited
}

\author{
Mio Fukuhara ${ }^{\mathrm{a}}$, Ryota Misawa ${ }^{\mathrm{b}}$, Kazuki Niino ${ }^{\mathrm{a}}$, Naoshi Nishimura ${ }^{\mathrm{a}, *}$ \\ ${ }^{a}$ Graduate School of Informatics, Kyoto University, Kyoto 606-8501, Japan \\ ${ }^{b}$ Graduate School of Engineering, Kyoto University, Kyoto 615-8540, Japan
}

\begin{abstract}
This study considers the stability of time domain BEMs for the wave equation in 2D. We show that the question of stability of time domain BEMs is reduced to a nonlinear eigenvalue problem related to frequency domain integral equations. We propose to solve this non-linear eigenvalue problem numerically with the Sakurai-Sugiura method. After validating this approach numerically in the exterior Dirichlet problem, we proceed to transmission problems in which we find that some time domain counterparts of "resonance-free" integral equations in frequency domain lead to instability. We finally show that the proposed stability analysis helps to reformulate these equations to obtain stable numerical schemes.
\end{abstract}

Keywords: stability, time domain, BEM, transmission problems, eigenvalue problems

\section{Introduction}

Among various numerical methods for partial differential equations such as finite difference methods or finite element methods, etc., boundary element methods (BEMs) are often said to be advantageous in wave problems because they can be applied to scattering problems easily. It is certainly true that BEMs in frequency domain are easy to use, but the same does not necessarily apply to time domain methods. As a matter of fact, BEMs for

\footnotetext{
${ }^{*}$ Corresponding author

Email addresses: fukuhara@acs.i.kyoto-u.ac.jp (Mio Fukuhara), misawa.ryota.8c@kyoto-u.ac.jp (Ryota Misawa), niino@acs.i.kyoto-u.ac.jp (Kazuki Niino), nchml@i.kyoto-u.ac.jp (Naoshi Nishimura) 
the wave equation in time domain have a long standing stability problem and there have been many efforts to stabilise BEMs for wave equations. For example, Ha Duong and his colleagues (e.g., [1]) showed the stability of some time domain BEMs in 3D based on space-time variational (Galerkin) formulations. Their argument depends on the energy conservation which is why their variational formulation includes time derivatives (e.g., the time derivative of single layer potential). Aimi et al. presented some numerical results in 2D using time or space differentiated integral equations and a space-time variational approach. Abboud et al. [3] considered a coupling of space-time variational BEMs with discontinuous Galerkin methods. Unfortunately, however, implementing computational codes for the full space-time variational formulation is not very easy. Coding becomes easier if one uses variational approaches only spatially and use collocation in time. Van 't Wout et al. [4] have shown a way to find a stable time-collocated variational approach based on space-time variational methods. In spite of these efforts, the standard collocation approaches remain the preferred choice in engineering, although known mathematical stability results in collocation are rather limited (see Davies and Dancan [5] for example. We remark that this reference [5] is the only paper cited as an example of known stability results for time domain collocation BEM for hyperbolic problems in the related chapter in Encyclopedia of Computational Mechanics [6] indicating how little we know about this issue). Various numerical stabilisation techniques for collocation have been proposed, from which we cite just a few relatively new ones. Parot et al. discussed the removal of a non-oscillatory instability in a hypersingular integral equation[7] as well as the stabilisation of oscillatory ones by scaling [8]. Jang and Ih proposed to use the time domain version of CHIEF method and a filtering technique to stabilise BEMs for exterior problems[9]. They also consider stabilisation for interior problems using a filtering technique[10]. Pak and Bai[1] proposed a variable-weight multi-step collocation scheme with time projection in their regularised BEM for elastodynamics, which is combined with an eigenvalue analysis 12 . These numerical stabilisation techniques have been shown to be effective via numerical examples, although they require numerical solutions of large eigenvalue problems. For further literatures on numerical stabilisation techniques, we refer the reader to the lists of references of above mentioned papers. Some other investigations take viewpoints similar to ours in that they seek stabilisation based on the choices of integral equations. For example, the use of time differentiated integral equations has been advocated by several authors 13, 14. Ergin et 
al. [15] proposed to use the Burton-Miller (BM) integral equation to achieve stability guided by an observation that the instability of BEMs for scattering problems is related to fictitious eigenfrequencies (internal resonance). Chappell et al.[16, 17] gave further insight as well as the implementation details of the BM formulation. This formulation has been utilised recently in practical applications 18. These approaches based on the choices of integral equations are of interest because they are directly related to the cause of instability thus providing intuition for the stabilisation strategies, albeit qualitatively. Finally we mention recent developments of CQM by Lubich[19, 20, 21] which is a stable method of computing convolutions. CQM has been applied successfully to engineering applications (e.g., Schanz et al. [22]). However, implementing CQM is still not as simple as the standard collocation methods, which is the reason we consider the conventional approach in this paper.

The above brief review of the works on the stability of time domain BEMs for the wave equation covers just a small part of what have been done so far. Indeed, the cause of the instability is now fairly well understood in connection with the spectra of the integral operators and the error introduced by discretisation (e.g., [15, 16, 23]), particularly in exterior problems. In spite of these efforts by predecessors, however, there seem to exist no definite and simple criteria of stability for the collocation methods. One still needs to carry out a quantitative assessment numerically in order to see if a particular scheme is stable or not. A standard method to check the stability of collocation BEMs in time domain is to compute characteristic roots by solving a polynomial eigenvalue problem (see (11)) after reducing it to Walker et al.[24]). This method is effective in 3D where the fundamental solution has a finite "tail" (i.e., it vanishes after a finite time). However, this approach needs linear eigensolvers for sparse, but large, matrices. One may possibly solve polynomial eigenvalue problems directly to reduce the size of the matrix, but this will lead to a non-linear eigenvalue problem. Fortunately, recent developments of eigensolvers based on contour integrals such as the Sakurai-Sugiura method (SSM) 25] made the solution of non-linear eigenvalue problems feasible. In $2 \mathrm{D}$ problems, however, the same approach is not very practical because the fundamental solution in $2 \mathrm{D}$ is very slow to decay in time. In this paper we propose to resolve this difficulty by carrying out the required stability analysis in frequency domain. Namely, we convert the stability analysis for BEMs in two dimensional wave equation to a non-linear eigenvalue problem similar to those for the Helmholtz equation and solve it 
with SSM using techniques proposed in Misawa et al. [26, 27]. The bene-

fit of this approach is twofold. Firstly, this method provides an alternative to the standard stability analysis in time domain. The proposed approach is applicable to $2 \mathrm{D}$ problems without ambiguity and remains valid in $3 \mathrm{D}$ problems as well. In contrast to this the standard time domain approach is not applicable to 2D problems in an unequivocal manner. Secondly, the proposed approach has an additional benefit of providing new intuition into the subject. Specifically, it is useful in selecting stable and highly accurate integral equations in various problems. Indeed, we shall use the proposed method to investigate the stability of various time domain integral equations for transmission problems, which have not been investigated very much so

As a basic study in this subject, however, this paper considers only very simple problems as examples of the use of the proposed stability analysis. Namely, we restrict our attention mainly to exterior Dirichlet problems and transmission problems for domains bounded by a circle. We first present a stability analysis for the exterior Dirichlet problem using frequency domain tools. The question of stability is then reduced to the computation of the characteristic roots which are eigenvalues of a certain non-linear equation. After solving this eigenvalue problem with SSM ignoring the effect of the spatial discretisation, we identify potentials which yield stable numerical schemes with piecewise linear time basis functions and a particular choice of discretisation parameters. We then proceed to transmission problems in which we show that even the time domain counterparts of "resonance free" BEMs may lead to instability. To stabilise these integral equations we modify them using potentials which have been concluded to yield stable numerical schemes. We show that numerical schemes derived from these modified formulations do lead to stability via numerical experiments in time domain as well as our stability analysis. After examining the influence of the spatial discretisation on the characteristic roots, we present numerical examples of transmission problems for non-circular domains solved with the modified integral equations, which appear to be stable.

\section{Exterior Dirichlet problems}

\subsection{Formulation}

Let $D_{2} \subset \mathbb{R}^{2}$ be a bounded domain whose boundary $\Gamma=\partial D_{2}$ is smooth and let $D_{1}$ be the exterior of $D_{2}$, i.e., $D_{1}=\mathbb{R}^{2} \backslash \bar{D}_{2}$. Also, let $n$ be the unit 
normal vector on $\Gamma$ directed towards $D_{1}$. We are interested in the following initial- boundary value problem (Dirichlet problem):

Find $u$ which satisfies the two dimensional wave equation in $D_{1}$ :

$$
\Delta u-\frac{1}{c_{1}^{2}} \frac{\partial^{2} u}{\partial t^{2}}=0 \quad \text { in } D_{1} \times(t>0)
$$

the homogeneous Dirichlet boundary condition on $\Gamma$ for $u$ :

$$
u=0 \quad \text { on } \Gamma \times(t>0)
$$

the homogeneous initial conditions in $D_{1}$ :

$$
\left.u^{\mathrm{sca}}\right|_{t=0}=\left.\frac{\partial u^{\mathrm{sca}}}{\partial t}\right|_{t=0}=0, \quad \text { in } D_{1}
$$

and the radiation condition for the scattered wave $u^{\text {sca }}=u-u^{\text {inc }}$ in $D_{1}$, where $c_{1}$ is the wave speed in $D_{1}$ which is written as $c_{1}=\sqrt{\frac{s_{1}}{\rho_{1}}}, s_{1}$ and $\rho_{1}$ are the shear modulus and density in $D_{1}$ and $u^{\text {inc }}$ is the incident wave which satisfies (1) in the whole space-time, respectively.

\subsection{Boundary integral equations}

The solution to the above initial- boundary value problem can be written as

$$
u(x, t)=u^{\text {inc }}(x, t)-S^{1} q(x, t) \quad(x, t) \in D_{1} \times(t>0)
$$

if the function $q(x, t)$ on $\Gamma \times(t>0)$ is chosen such that

$$
0=u^{\text {inc }}(x, t)-S^{1} q(x, t) \quad(x, t) \in D_{2} \times(t>0)
$$

is satisfied, where $S^{\nu}(\nu=1$ or $2 . \nu=1$ in the present context) stands for the single layer potential defined by

$$
S^{\nu} q(x, t):=\int_{0}^{t} \int_{\Gamma} G^{\nu}(x-y, t-s) q(y, s) d S_{y} d s
$$

and $G^{\nu}(x, t)$ is the fundamental solution of the wave equation given by:

$$
G^{\nu}(x, t)=\frac{c_{\nu}}{2 \pi \sqrt{\left(c_{\nu} t\right)^{2}-|x|^{2}}+}= \begin{cases}\frac{c_{\nu}}{2 \pi \sqrt{\left(c_{\nu} t\right)^{2}-|x|^{2}}} & c_{\nu} t>|x| \\ 0 & \text { otherwise. }\end{cases}
$$


For later convenience, we also introduce the normal derivative of the single layer potential $D^{T \nu}$, the double layer potential $D^{\nu}$ and its normal derivative $N^{\nu}$ defined by:

$$
\begin{aligned}
D^{T \nu} q(x, t) & :=\int_{0}^{t} \int_{\Gamma} \frac{\partial G^{\nu}}{\partial n_{x}}(x-y, t-s) q(y, s) d S_{y} d s \\
D^{\nu} q(x, t) & :=\int_{0}^{t} \int_{\Gamma} \frac{\partial G^{\nu}}{\partial n_{y}}(x-y, t-s) q(y, s) d S_{y} d s \\
N^{\nu} q(x, t) & :=\int_{0}^{t} \int_{\Gamma} \frac{\partial^{2} G^{\nu}}{\partial n_{x} \partial n_{y}}(x-y, t-s) q(y, s) d S_{y} d s .
\end{aligned}
$$

By these notations for potentials we indicate functions defined in $x \in \mathbb{R}^{2} \backslash$ $\Gamma$ in this paper. Their boundary traces on $\Gamma$ from the exterior (interior) are indicated by superposed $+(-)$. When the exterior and interior traces coincide, however, we denote them by the same letter without superposed \pm . This applies to $S^{\nu}, \dot{S}^{\nu}$ and $N^{\nu}$, but we need to evaluate integrals in $N^{\nu}$ in the sense of the finite part then.

The function $q(x, t)$ coincides with the (exterior trace of) normal derivative of $u$ on $\Gamma$ if (3) is satisfied. The condition in (3) leads to several boundary integral equations defined on the boundary of the scatterer. Four of standard boundary integral equations on $\Gamma \times(t>0)$ are given as follows:

$$
\begin{aligned}
& u^{\text {inc }}(x, t)-S^{1} q(x, t)=0 \\
& \dot{u}^{\text {inc }}(x, t)-\dot{S}^{1} q(x, t)=0 \\
& \frac{\partial u^{\text {inc }}}{\partial n}(x, t)-\left(D^{T 1}\right)^{-} q(x, t)=0 \\
& \frac{\partial u^{\text {inc }}}{\partial n}(x, t)+\frac{1}{c_{1}} \dot{u}^{\text {inc }}(x, t)-\left(D^{T 1}\right)^{-} q(x, t)-\frac{1}{c_{1}} \dot{S}^{1} q(x, t)=0
\end{aligned}
$$

where 'stands for the time derivative. Equations of these types have been considered by many authors for various potentials mainly in 3D. Indeed, (5) is the ordinary BIE. The time differentiated equation in (6) has been considered in [13]. Bamberger and Ha Duong[1] also discussed a space-time variational version of this equation in 3D. Equation similar to (7) for the double layer potential in 3D has been utilised by Parot et al. [7, 8] while (8) for the double layer potential in 3D has been considered in Ergin et al.[15] 140 and Chappell et al. [16] among others. The coupling constant in (8) seems to be the most natural choice because (8) is then derived as one imposes the 
first order approximation of the absorbing boundary condition [28] on $\Gamma$ to the RHS (right hand side) of (3), thus "exteriorising" the interior domain $D_{2}$. The discussion in Chappell and Harris 17 also seems to support this

\subsection{Stability}

We consider the following Volterra integral equation which is typically a time domain BIE obtained by discretising (5)-(8) in the spatial direction by using collocation:

$$
f(t)=\int_{0}^{t} K(t-s) v(s) d s
$$

where $K$ represents an $N \times N$ matrix and $v$ and $f$ stand for unknown and given $N$-vectors, respectively. Note that $K$ may include terms of the form $c \delta(\cdot)$ or its derivatives, where $c$ is a constant and $\delta(\cdot)$ is Dirac's delta functions. Discretising the unknown function $v(s)$ in (9) using time interpolation functions $\phi_{m}(s)$ as $v(s) \approx \sum_{m} \phi_{m}(s) v_{m}$, we obtain the following algebraic equation:

$$
\begin{aligned}
f(l \Delta t) & =\sum_{m=1}^{l} \int_{0}^{l \Delta t} K(l \Delta t-s) \phi_{m}(s) d s v_{m} \\
\phi_{m}(s) & =\phi_{\Delta t}(s-m \Delta t)
\end{aligned}
$$

where $\phi_{\Delta t}(t)$ is a basis function which satisfies $\phi_{\Delta t}(k \Delta t)=1(k=0)$ or $\phi_{\Delta t}(k \Delta t)=0(k \neq 0)$ for $k \in \mathbb{Z}, \Delta t$ is the time increment, $l$ is the number of time steps, respectively. Usually, an algebraic equation in the form of 10 is solved in a time marching manner for $v_{m}(m=1,2, \cdots)$ in time domain BEMs.

Obviously, the stability of the resulting numerical scheme is a concern in solving BIEs in time domain. To examine this issue, we follow the standard argument 24] to put $v_{m}=\lambda^{m} v$ in the homogeneous version of 10 where $\lambda \in \mathbb{C}$ is a number and $v$ is an element of $\mathbb{C}^{N}$. This gives

$$
0=\sum_{m=0}^{l-1} \int_{0}^{l \Delta t} K(s) \phi_{\Delta t}(m \Delta t-s) d s \lambda^{-m} v
$$


Suppose $l$ is taken sufficiently large. A complex number $\lambda$ is said to be an eigenvalue of (11) if there exists a non-trivial $v$ which satisfies (11). Then our definition of the stability is the following: the scheme is stable if all the eigenvalues of (11) satisfy $|\lambda| \leq 1$. The scheme is unstable if there exists an eigenvalue of (11) s.t. $|\lambda|>1$ holds. Eigenvalue problems of this type in 3D have been considered by many authors after converting them into equivalent linear eigenvalue problems for the companion matrices (e.g., [24, 23, 8, 10, 4, 12,). As a matter of fact, there is no ambiguity in the choice of a sufficiently large $l$ in 3D if the scatterer is bounded because the fundamental solution has a "tail" of a finite length. In 2D problems, however, this is not the case since the tail of the fundamental solution has an infinite length, as one sees in (4). In addition, the time decay of the fundamental solution is slow, thus making it difficult to set an appropriate truncation number $l$ in (11).

To proceed further, we put $\lambda=e^{-i \Omega \Delta t}$ where $\Omega$ is a complex number. The stability criterion is now rewritten as follows: $\operatorname{Im} \Omega \leq 0(\operatorname{Im} \Omega>0)$ implies stability (instability). Also, suppose that $\phi_{\Delta t}(s)=0$ for $s<-\Delta t$; a condition satisfied by many choices of the basis function including a piecewise linear one. We then let $l$ tend to infinity in $(11)$ to have

$$
0=\sum_{m=-\infty}^{\infty} \int_{0}^{\infty} K(s) \psi_{\Delta t}(s-m \Delta t) e^{i m \Delta t \Omega} d s v
$$

where $\psi_{\Delta t}(s)=\phi_{\Delta t}(-s)$, which is nothing other than the discretised Fourier transform of $K$. Obviously, this expression approximates the Fourier transform $\hat{K}$ of $K$ precisely in lower frequencies, but just roughly in higher frequencies. This suggests a connection between the stability of the time domain BEM and the eigenvalues of the frequency domain BEM; an observation made by many authors (e.g., [15, 16]).

We now write $K$ in terms of $\hat{K}$ as

$$
K(s)=\frac{1}{2 \pi} \int_{-\infty}^{\infty} \hat{K}(\omega) e^{-i \omega s} d \omega
$$

Using the Poisson summation formula, we rewrite 12 into

$$
0=\sum_{m=-\infty}^{\infty} \frac{1}{\Delta t} \hat{K}\left(\Omega_{m}\right) \hat{\phi}_{\Delta t}\left(\Omega_{m}\right) v, \quad \Omega_{m}=\Omega-\frac{2 m \pi}{\Delta t}
$$


where $\hat{\phi}_{\Delta t}$ is the Fourier transform of $\phi_{\Delta t}$ which is given by

$$
\hat{\phi}_{\Delta t}(\Omega)=\frac{2}{\Omega^{2} \Delta t}(1-\cos \Omega \Delta t)
$$

for the particular case of the piecewise linear $\phi_{\Delta t}$. The stability issue of the time domain BEM is thus reduced to a non-linear eigenvalue problem of finding $\Omega \in \mathbb{C}$ with which 13 has a non-trivial solution $v \in \mathbb{C}^{N}$. Hence, we call these eigenvalues $\Omega$ 's as the characteristic roots of $(13)$. We note that the expression on the right hand side of $(13)$ is periodic with respect to $\Omega$ with the period of $2 \pi / \Delta t$. We also note that the present formulation assumes the use of time basis functions and a constant $\Delta t$. Therefore, it does not apply to formulations which do not use time elements/meshes or to variable time steps.

We now consider the limit of $\Delta t \rightarrow 0$ in (13) in a somewhat intuitive manner. More rigorous arguments could be made with particular choices of kernel and basis functions. We first note that

$$
\frac{\phi_{\Delta t}(s)}{\Delta t} \rightarrow \delta(s) \text { and } \frac{\hat{\phi}_{\Delta t}}{\Delta t} \rightarrow 1
$$

hold as $\Delta t \rightarrow 0$ if $\phi_{\Delta t}$ can interpolate a constant function exactly. Hence, we expect to have

$$
\sum_{m=-\infty}^{\infty} \frac{1}{\Delta t} \hat{K}\left(\Omega_{m}\right) \hat{\phi}_{\Delta t}\left(\Omega_{m}\right) \rightarrow \hat{K}(\Omega) \quad \text { as } \Delta t \rightarrow 0
$$

if $\hat{K}(\Omega) \rightarrow 0$ as $|\Omega| \rightarrow \infty$, which is the case in 2D. From this result, we expect that the characteristic roots of (13) are obtained as perturbations of the eigenvalues of the corresponding frequency domain BIEs. We note that a similar observation has been made in Chappell et al.[16, 17] qualitatively.

It is well-known that the eigenvalues of the frequency domain BIE can be classified into true and fictitious eigenvalues [26, 27]. In the exterior problems, the true eigenvalues are with negative imaginary parts, while the behaviour of the fictitious eigenvalues depend on the particular choice of integral equations. In (5) - (7) the fictitious eigenvalues of the corresponding frequency domain BIEs are real valued, while those of (8) are with negative imaginary parts. It is therefore natural to expect that equations (5)-(7) are more prone to instability than (8). However, (5)-(7) may still turn out to be stable after discretisation depending on the choice of the time basis function because real 
eigenvalues of the frequency domain BIE may move to lower complex plane

after the time discretisation. Also, (8) may turn out to be unstable if the perturbation of the eigenvalues is very large.

\subsection{Simplified stability analysis for circular domains}

One may use methods based on contour integrals such as the SakuraiSugiura Method (SSM) in the solution of non-linear eigenvalue problem in (13) for a general boundary $\Gamma$. Indeed, one may replace

$$
\hat{G}^{\nu}(x)=\frac{i}{4} H_{0}^{(1)}\left(\Omega|x| / c^{\nu}\right)
$$

in the Fourier transformed versions of BIEs in (5)-(8) by

$$
\frac{i}{4} \sum_{m=-\infty}^{\infty} H_{0}^{(1)}\left(\Omega_{m}|x| / c^{\nu}\right) \frac{\hat{\phi}_{\Delta t}\left(\Omega_{m}\right)}{\Delta t}
$$

to this end, where $H_{0}^{(1)}$ is the Hankel function of the 1st kind. In the present paper, however, we shall pay attention to a simple special case in which $\Gamma$ is a unit circle. Also, we restrict out attention to the piecewise linear time basis functions for the purpose of simplicity.

We consider (13) for a unit circle $\Gamma$ without spatial discretisation (the effect of the spatial discretisation will be discussed later). In this case we can simplify the non-linear eigenvalue problem in (13) using the Fourier series with respect to the angular variable. Indeed, we use the well-known Graf addition theorem[29] to have

$$
\begin{array}{r}
\hat{G}(x-y)=\frac{i}{4} \sum_{n=-\infty}^{\infty} H_{n}^{(1)}(k|x|) J_{n}(k|y|) e^{i n(\Theta-\theta)}, \\
x=|x|(\cos \Theta, \sin \Theta), \quad y=|y|(\cos \theta, \sin \theta)
\end{array}
$$

when $|x|>|y|$ holds, where $J_{n}$ is the Bessel function, $k=\Omega / c$ and $\Theta$ and $\theta$ are the azimuth angles of $x$ and $y$. The role of $H_{n}^{(1)}$ and $J_{n}$ in $(16)$ is interchanged when $|x|<|y|$. In (16) we have suppressed the superfix $\nu$ for the domain in order to simplify the notation. From (13) and (16), we see that the characteristic roots of the time discretised boundary integral equations corresponding to (5)-(7) are obtained as zeros of the expressions 
in the following list:

$$
\begin{aligned}
& S \leftrightarrow \sum_{m} H_{n}^{(1)}\left(\Omega_{m} / c\right) J_{n}\left(\Omega_{m} / c\right) \hat{\phi}_{\Delta t}\left(\Omega_{m}\right) \\
& \dot{S} \leftrightarrow f_{1}(\Omega ; n, c)=-\sum_{m} i \Omega_{m} H_{n}^{(1)}\left(\Omega_{m} / c\right) J_{n}\left(\Omega_{m} / c\right) \hat{\phi}_{\Delta t}\left(\Omega_{m}\right) \\
& D^{T-}\left(, D^{+}\right) \leftrightarrow f_{2}(\Omega ; n, c)=\sum_{m} \Omega_{m} / c H_{n}^{(1)}\left(\Omega_{m} / c\right) J_{n}^{\prime}\left(\Omega_{m} / c\right) \hat{\phi}_{\Delta t}\left(\Omega_{m}\right)
\end{aligned}
$$

where $n$ is an integer between 0 and a large number $n_{\max }$. The characteristic equation for $(8)$ is obtained from (18) and $(19)$ as

$$
\sum_{m} H_{n}^{(1)}\left(\Omega_{m} / c\right) \Omega_{m} / c\left(J_{n}^{\prime}\left(\Omega_{m} / c\right)-i J_{n}\left(\Omega_{m} / c\right)\right) \hat{\phi}_{\Delta t}\left(\Omega_{m}\right) .
$$

Note that the series on the right hand sides of eqs. (17)- 20 are absolutely convergent.

\subsection{Numerical experiments}

We now carry out numerical experiments to see if the stability analysis given in the previous section can predict the behaviour of the time domain BEM correctly.

To this end, we consider the problem defined in 2.1 where the boundary $\Gamma$ is the unit circle. The material constants are $s_{1}=\rho_{1}=c_{1}=1$. The incident wave is a plane wave given by:

$$
u^{\text {inc }}= \begin{cases}0 & \left(c_{1} t-x_{1}-t_{0} \leq 0\right) \\ \frac{\left(c_{1} t-x_{1}-t_{0}\right)^{2}}{2} & \left(c_{1} t-x_{1}-t_{0}>0\right)\end{cases}
$$

where $t_{0}=1+2 \Delta t$. We use piecewise constant boundary elements, piecewise linear temporal elements and the collocation method to discretise the BIEs in (5)-(8). All the required integrals are computed exactly. The boundary is discretised into 100 elements, the time increment is set as $\Delta t=\frac{2 \pi}{100}$ and the number of time steps is 1000. Also, the characteristic roots of (13) are calculated with (17)-(20) and SSM.

Figs 1(a) 1(d) show the results obtained with (5)-(8), respectively. We plot $q$ for every 10 time steps in these figures (this applies to all subsequent time domain results). Also, Fig,2 gives the "exact" solution obtained numerically with the frequency domain exact solution and FFT. We see that the 
standard BIE in (5) is unstable, and the time derivative BIE in (6) and the time domain BM BIE in (8) are stable. The normal derivative BIE in (7) does not show divergence, but deviates considerably from the "exact" solution. The BM result is not as bad as the normal derivative one, but is seen to drift from the exact solution by a time dependent constant. The accuracy of the time derivative BIE appears to be satisfactory.

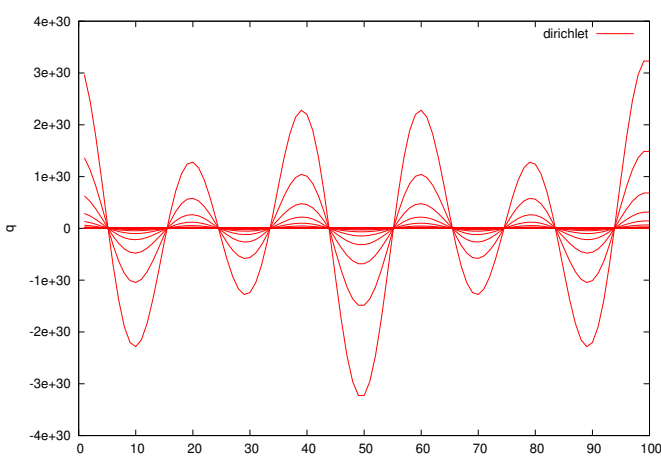

(a) Eq. (5)

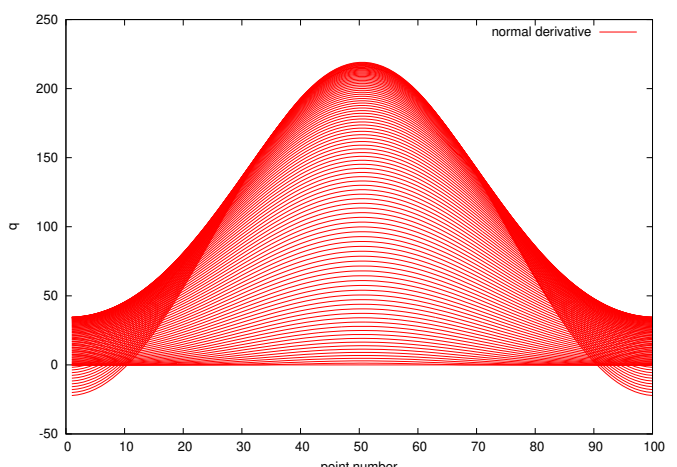

(c) Eq. (7)

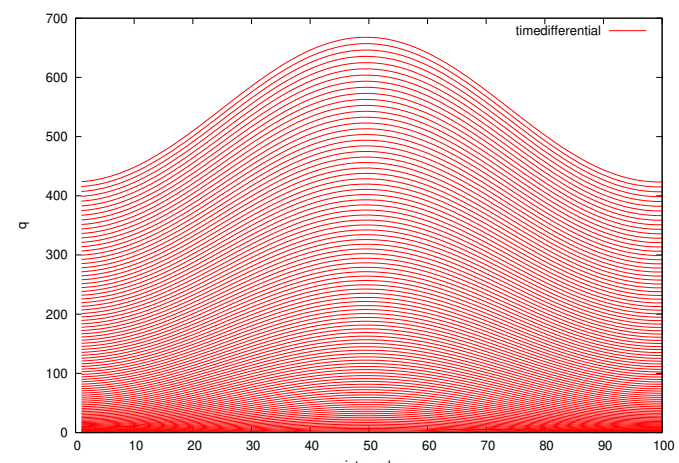

(b) Eq. (6)

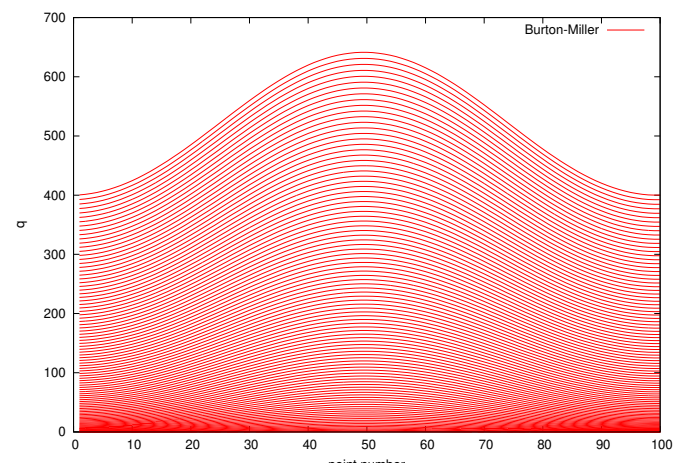

(d) Eq. (8)

Figure 1: $q$ obtained with various integral equations vs point number. The point number and the azimuth angle $\theta$ are related by $\theta=-2 \pi / 100 \times$ (point number $+1 / 2$ )

We next check the behaviour of the characteristic roots of these time domain BIEs using SSM. We set the range for computing eigenvalues to be $0 \leq \operatorname{Re} \Omega \leq 50=\frac{\pi}{\Delta t}$ and $-2 \leq \operatorname{Im} \Omega \leq 2$ considering the periodicity of 113 and the fact that the characteristic roots are located symmetrically with respect to the imaginary axis, which can be easily shown using the explicit 


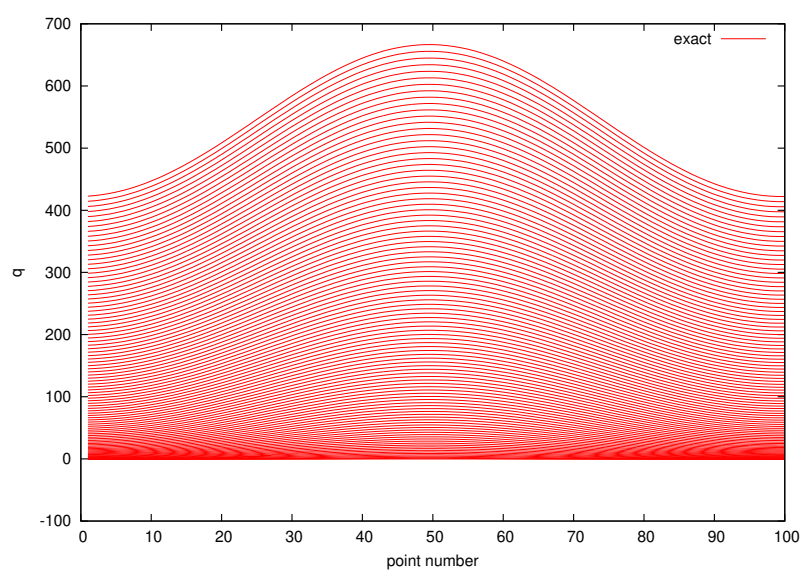

Figure 2: "Exact" solution of $q$

forms of (13). Note that the upper limit of $\Omega$ is consistent with the Nyquist frequency associated with $\Delta t$. Also, we took $n_{\max }$ to be 60 considering the number of boundary subdivisions and the spatial Nyquist "frequency". In the computation, we redefine the Hankel functions so that they have branch cuts on the negative imaginary axis rather than on the negative real axis. This guarantees that the expression in $(13)$ is analytical when $0<\operatorname{Re} \Omega<\frac{\pi}{\Delta t}$ holds.

Figs 3(a)-Fig 3(d) show the characteristic roots of the BIEs in (5)-(8), respectively. We plot the eigenvalues of (13) for various BIEs (i.e., zeros of the expressions in (17)-(20) $)$ in green and the eigenvalues of the frequency domain BIEs given by $\hat{K}(\Omega) v=0$ (i.e., zeros of the products of Hankel and Bessel functions obtained by setting $m=0$ in $(17)-(20))$ in red. Note that the red symbols near the imaginary axis in Figs $3(\mathrm{a})-\mathrm{Fig} 3(\mathrm{~d})$ are the true eigenvalues for the exterior Dirichlet problem, while those on the real axis are fictitious ones related to the interior Dirichlet problems in Figs.3(a) and $3(\mathrm{~b})$ and to the Neumann problem in Fig.3(c). The fictitious eigenvalues for (8) are those associated with interior impedance boundary value problems. We note that all BIEs in (5)-(8) have characteristic roots close to the true or fictitious eigenvalues, but other characteristic roots are scattered and quite far from any of eigenvalues of the corresponding frequency domain BIEs, except in the BM equation in (8).

250 From these figures, we see that the BIE (5) has the characteristic roots 


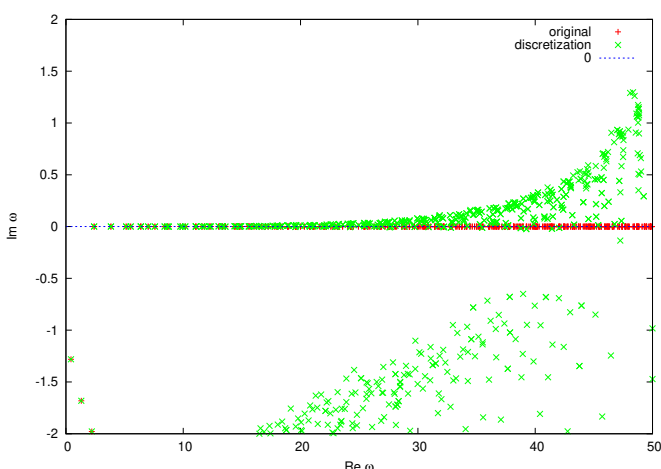

(a) Eq. (5)

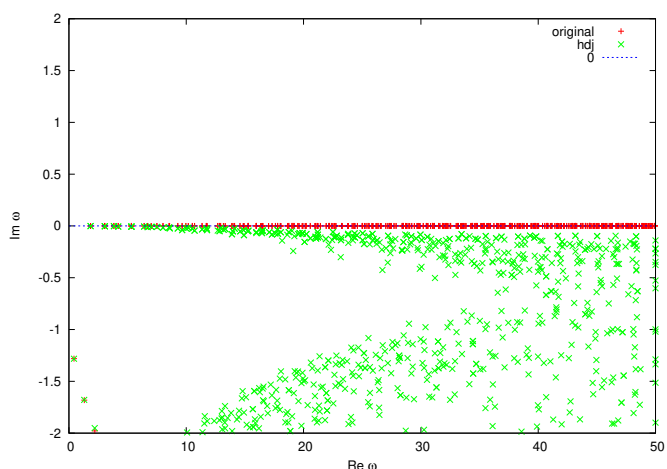

(c) Eq. (7)

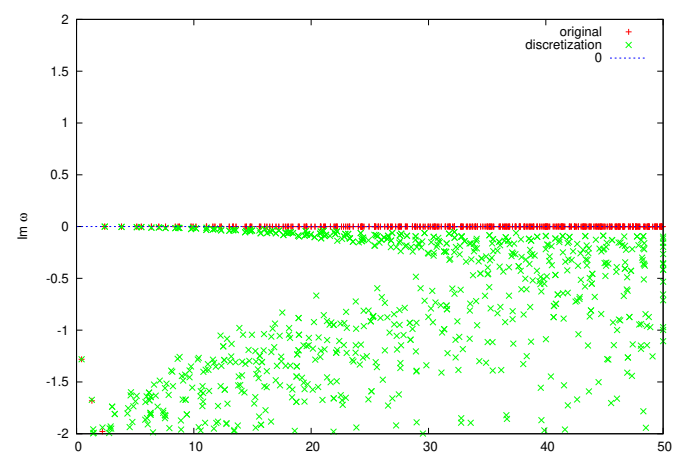

(b) Eq. (6)

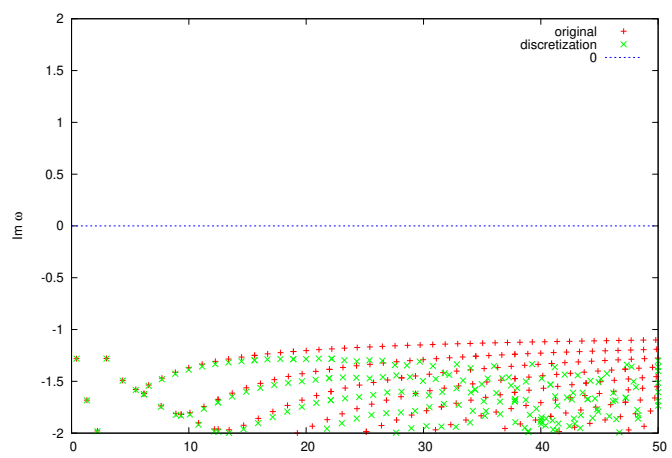

(d) Eq. 8

Figure 3: Characteristic roots of various integral equations. $\times$ : characteristic roots, + : eigenvalues of frequency domain BIEs

with positive imaginary parts, but this is not the case with other BIEs. These results are consistent with the corresponding time domain results in Fig. 1. Also, the inaccuracy of (7) is considered to be related to the fact that $\Omega=0$ is an eigenvalue of $(19)$ with $n=0$. We remark that a similar case has been reported in Parot et al. [7] where this phenomenon has been called a "pneumatic mode". As a matter of fact $\Omega=0$ is a zero of both (18) (for all $n$ ) and (20) (for $n=0$ ) as well. An adverse effect of this eigenvalue on (8) is visible in the constant shift of the solution in Fig.1(d) (compare with Fig,2), although not as evidently as in Fig, 1(c).

To examine the effect of this zero eigenvalue on the numerical solution of 
(6), we consider another incident wave given by

$$
u^{\text {inc }}=\left\{\begin{array}{ll}
0 & \left(c_{1} t-x_{1}-t_{0} \leq 0\right) \\
\frac{\left(c_{1} t-x_{1}-t_{0}\right)^{2}}{c_{1} t-x_{1}-t_{0}+4 \Delta t} & \left(c_{1} t-x_{1}-t_{0}>0\right)
\end{array},\right.
$$

260

which is a smoothed linear function, where $t_{0}=1+2 \Delta t$. Setting other parameters the same as in the previous example, we solve (6) to compute the time history of $q$ as plotted in Fig $4(\mathrm{a})$. Comparing this result with the "exact" solution given in Fig, $4(\mathrm{c})$ one sees that an error having a zigzag pattern is superimposed on the solution of (6). This is in contrast to the BM solution given in Fig 4(b) which is smooth, but not accurate. This result can be explained as follows. With (6), the (spatially) high frequency error incurred initially by the mismatch of the wavefront and mesh remains undamped after a long time because of the existence of a zero characteristic root with high $n$ eigenfunctions. Since this eigenvalue is zero, this error does not propagate, decay or amplify. In other words, it persists. This type of error is included also in Fig 1(b), although its magnitude is too small to be visible. From these numerical experiments, we conclude that none of the integral equations in (5)-(8) are satisfactory!

A possible remedy for all these problems is to use an integral equation given by

$$
\begin{aligned}
\frac{\partial u^{\mathrm{inc}}}{\partial n}(x, t) & +\frac{1}{c_{1}} \dot{u}^{\mathrm{inc}}(x, t)+\alpha u^{\mathrm{inc}}(x, t) \\
& -\left(D^{T 1}\right)^{-} q(x, t)-\frac{1}{c_{1}} \dot{S}^{1} q(x, t)-\alpha S^{1} q(x, t)=0,
\end{aligned}
$$

which is the time domain counterpart of the BM equation with a complex (not pure imaginary) coupling constant, where $\alpha$ is a (real) number. It is easy to see that $\Omega=0$ is not a characteristic root of this equation. The numerical solution $q$ obtained with $(23)$ and the incident wave in $(22)$ is given in Fig 5(a) and its characteristic roots are shown in Fig, $5(\mathrm{~b})$, where we set $\alpha=1$. The high accuracy and stability of this formulation is evident from these figures, thus showing the usefulness of the proposed stability analysis in the selection of integral equations. See appendix for further numerical experiments for (23).

Epstein et al. 30] investigated time domain integral equations whose solutions exhibit correct long-time behaviours. From this viewpoint, (23) seems 


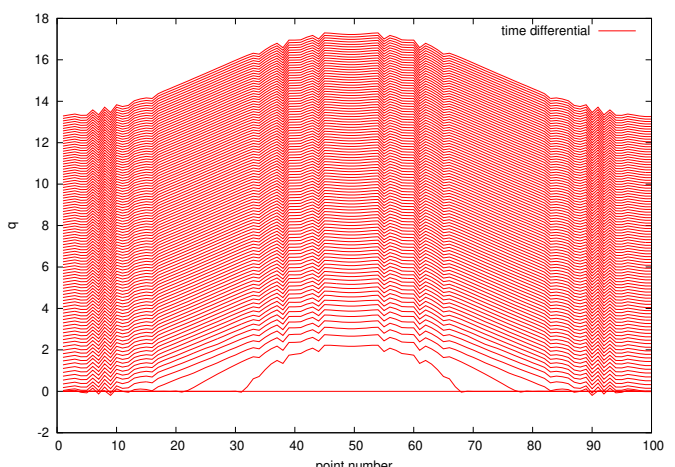

(a) Eq. (6)

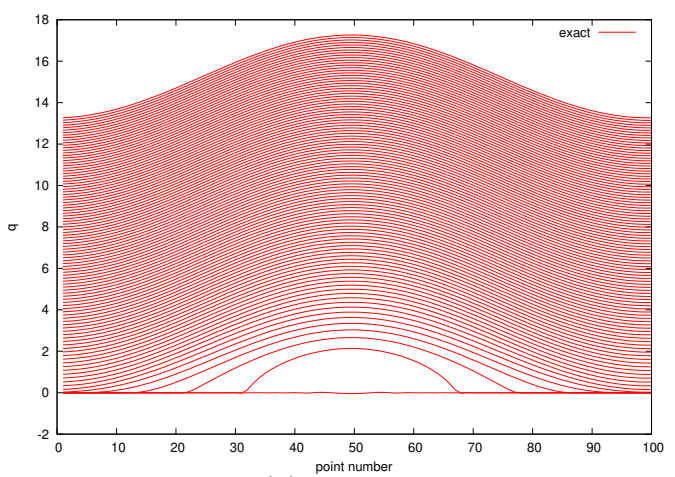

(c) "exact"

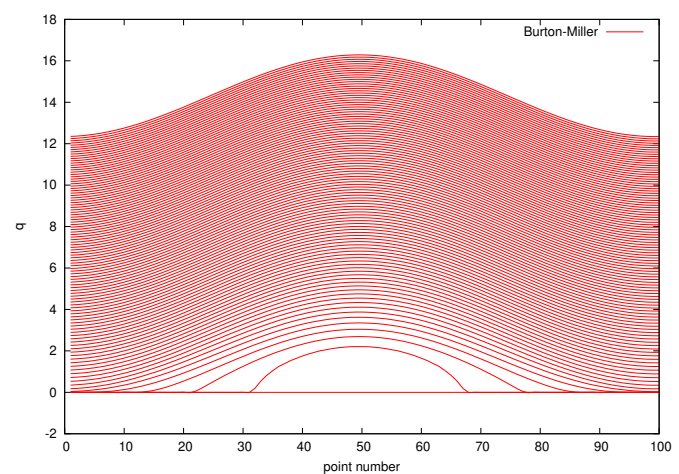

(b) Eq. (8)

Figure 4: $q$ obtained with various integral equations

to be a better choice than other stable choices in (6)-(8), although the characteristic root of (23) whose imaginary part has the minimum magnitude is close to one of fictitious eigenvalues (the one whose imaginary part is approximately equal to -0.8 in Fig. 5(b) rather than a true one.

\section{3. "Stable potentials"}

Motivated by the results in the previous section, we examine the stability of integral equations on the unit circle derived from potentials which may appear in BIEs. These potentials include the single layer $S\left(=S^{+}=S^{-}\right)$, traces of the normal derivatives of $S$ denoted by $D^{T \pm}$, the time derivative of $S$ denoted by $\dot{S}\left(=\dot{S}^{+}=\dot{S}^{-}\right)$and the traces of the double layer $D^{ \pm}$. Although we are also interested in the normal derivative of $D$ denoted by $N$, 


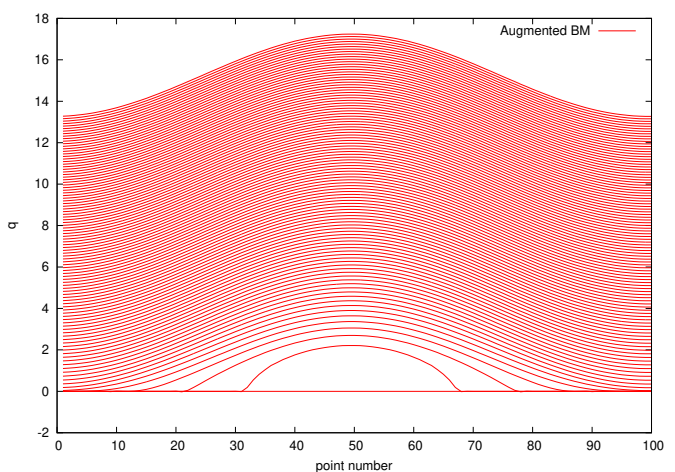

(a) $q$ obtained with eq. 23 .

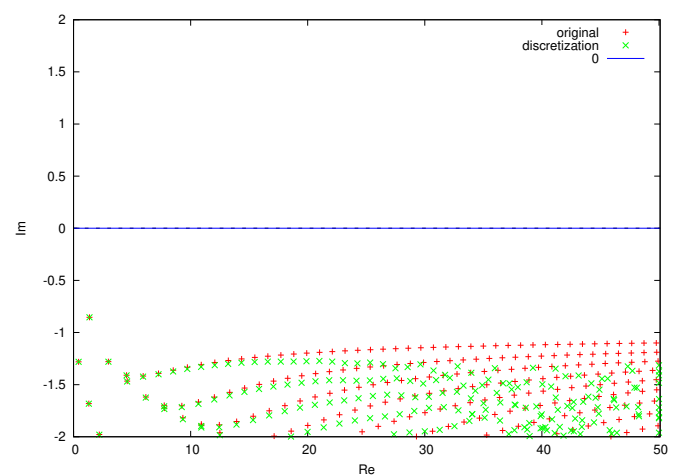

(b) Characteristic roots of $(23)(\times)$ and eigenvalues of the frequency domain BIE $(+)$

Figure 5: Augmented BM integral equation

it turned out that the simplified approach presented in 2.4 using the Fourier series expansion is not very easy to apply to $N$ with piecewise linear time basis functions because the series similar to (17)-(19) for $N$ does not converge absolutely (A similar observation applies to $D^{ \pm}$as well). Using a smoother time basis function could be a solution. As we shall see later, however, the time integrated normal derivative of the double layer potential defined by

$$
\begin{array}{r}
M u=\int_{0}^{t} \int_{\Gamma} \frac{\partial}{\partial n_{x}} \frac{\partial}{\partial n_{y}} \log \frac{c(t-s)+\sqrt{c^{2}(t-s)^{2}-|x-y|^{2}}+{ }_{+}}{|x-y|} u(y, s) d S_{y} d s \\
x \in \mathbb{R}^{2} \backslash \Gamma, \quad t>0
\end{array}
$$

is more useful than $N$ as far as the stability is concerned. We therefore carry out the stability analysis in 2.4 with $M\left(=M^{+}=M^{-}\right)$instead of $N$. Since the results for $S, \dot{S}$ and $D^{T-}=D^{+}$have already been given in Figs $3(\mathrm{a}) 3(\mathrm{c})$, we present those for $D^{T+}=D^{-}$and $M$ in Figs 6(a) 6(b) using the same material constants and time increment as before (i.e., $s_{1}=\rho_{1}=c_{1}=1$, $\Delta t=2 \pi / 100)$. They are zeros of the following expressions.

$$
\begin{aligned}
& D^{-}, D^{T+} \leftrightarrow f_{3}(\Omega ; n, c)=\sum_{m} \Omega_{m} / c H_{n}^{(1)^{\prime}}\left(\Omega_{m} / c\right) J_{n}\left(\Omega_{m} / c\right) \hat{\phi}_{\Delta t}\left(\Omega_{m}\right) \\
& M \leftrightarrow f_{4}(\Omega ; n, c)=\sum_{m} i \Omega_{m} / c^{2} H_{n}^{(1)^{\prime}}\left(\Omega_{m} / c\right) J_{n}^{\prime}\left(\Omega_{m} / c\right) \hat{\phi}_{\Delta t}\left(\Omega_{m}\right)
\end{aligned}
$$




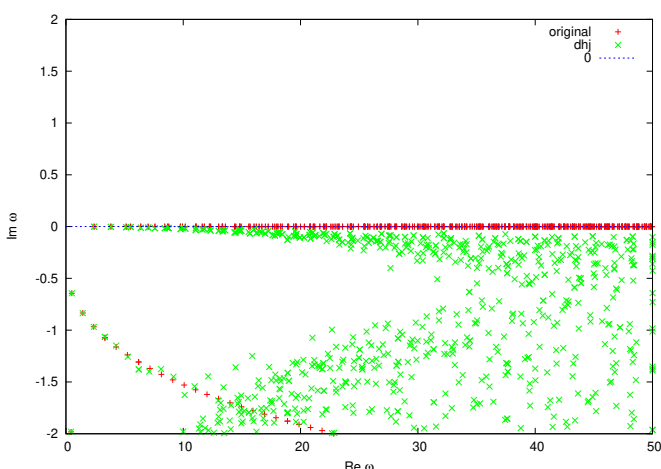

(a) $D^{T+}=D^{-}$

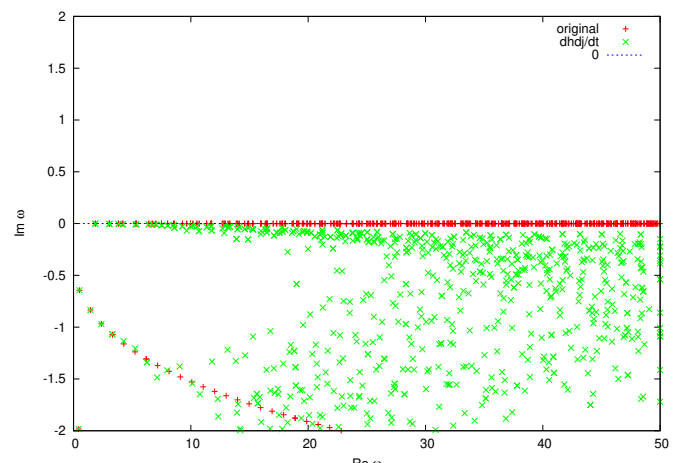

(b) $M$

Figure 6: Characteristic roots of various integral operators. $\times$ : characteristic roots, + : eigenvalues of frequency domain integral operators

From these results, we confirm that the equations obtained by discretising the following integral equations are numerically stable with piecewise linear time basis functions and parameters tested: (a) the time derivative of the single layer potential (b) the interior and exterior traces of the normal derivative of the single layer potential (c) the interior and exterior traces of the double layer potential (d) the time integrated normal derivative of the double layer potential. In the rest of this paper we call them "stable potentials" just for the purpose of simplicity. Note, however, that we have no claim of stability of these potentials except in the cases tested here.

In the next section, we combine these potentials to obtain numerically stable formulations in transmission problems.

\section{Transmission problems}

We are now interested in finding $u$ which satisfies (1),

$$
\Delta u-\frac{1}{c_{2}^{2}} \frac{\partial^{2} u}{\partial t^{2}}=0 \text { in } D_{2} \times(t>0),
$$

the transmission boundary conditions on $\Gamma$ :

$$
u^{+}=u^{-}(=u), \quad s_{1} \frac{\partial u^{+}}{\partial n}=s_{2} \frac{\partial u^{-}}{\partial n}(=q) \quad \text { on } \Gamma \times(t>0)
$$


and the homogeneous initial conditions

$$
\left.u\right|_{t=0}=\left.\dot{u}\right|_{t=0}=0 \quad \text { in } D_{2}
$$

in addition to the homogeneous initial and radiation conditions for $u^{\text {sca }}$ in (2), where $c_{2}$ is the wave speed in $D_{2}$ given by $c_{2}=\sqrt{\frac{s_{2}}{\rho_{2}}}$ and $\left(s_{2}, \rho_{2}\right)$ are the

shear modulus and density in $D_{2}$, respectively. The superscript $+(-)$ stands for the trace to $\Gamma$ from $D_{1}\left(D_{2}\right)$, respectively.

\subsection{Boundary integral equations}

There exist various possibilities of integral equation for transmission problems on $\Gamma$, of which we consider the following four [26, 27]:

PMCHWT

$$
\left(\begin{array}{cc}
-\left(D^{1}+D^{2}\right) & \frac{1}{s_{1}} S^{1}+\frac{1}{s_{2}} S^{2} \\
-\left(s_{1} N^{1}+s_{2} N^{2}\right) & D^{T 1}+D^{T 2}
\end{array}\right)\left(\begin{array}{c}
u \\
q
\end{array}\right)=\left(\begin{array}{c}
u^{\mathrm{inc}} \\
s_{1} \frac{\partial u^{\mathrm{inc}}}{\partial n}
\end{array}\right)
$$

Müller

$$
\left(\begin{array}{cc}
\frac{s_{1}+s_{2}}{2}-\left(s_{1} D^{1}-s_{2} D^{2}\right) & S^{1}-S^{2} \\
-\left(N^{1}-N^{2}\right) & \frac{s_{1}+s_{2}}{2 s_{1} s_{2}}+\frac{1}{s_{1}} D^{T 1}-\frac{1}{s_{2}} D^{T 2}
\end{array}\right)\left(\begin{array}{c}
u \\
q
\end{array}\right)=\left(\begin{array}{c}
s_{1} u^{\text {inc }} \\
\frac{\partial u^{\text {inc }}}{\partial n}
\end{array}\right)
$$

Burton-Miller

$\left(\begin{array}{cc}\frac{1}{2 c_{1}} \frac{\partial}{\partial t}-\left(N^{1}+\frac{1}{c_{1}} \dot{D}^{1}\right) & \frac{1}{2 s_{1}}+\frac{1}{s_{1}} D^{T 1}+\frac{1}{c_{1} s_{1}} \dot{S}^{1} \\ -\frac{1}{2}-D^{2} & \frac{1}{s_{2}} S^{2}\end{array}\right)\left(\begin{array}{c}u \\ q\end{array}\right)=\left(\begin{array}{c}\frac{\partial u^{\mathrm{inc}}}{\partial n}+\frac{1}{c_{1}} \frac{\partial u^{\mathrm{inc}}}{\partial t} \\ 0\end{array}\right)$

standard

$$
\left(\begin{array}{cc}
\frac{1}{2}-D^{1} & \frac{1}{s_{1}} S^{1} \\
\frac{1}{2}+D^{2} & -\frac{1}{s_{2}} S^{2}
\end{array}\right)\left(\begin{array}{c}
u \\
q
\end{array}\right)=\left(\begin{array}{c}
u^{\text {inc }} \\
0
\end{array}\right)
$$

In these equations we write $D^{\nu}$ for $\left(D^{\nu+}+D^{\nu-}\right) / 2$ etc. in order to show the non-integral terms explicitly at the cost of an abuse of notation. We note that there exist several versions of Müller's formulations for the wave equation. We here use the one in which the singularities of single layer potentials cancel. 


\subsection{Stable formulations}

The boundary integral equations shown in the previous section can be rewritten easily in terms of "stable potentials" presented in section 3 with the help of time differentiation and integration by parts. Here are the results:

modified PMCHWT

$$
\left(\begin{array}{cc}
-\left(D^{1}+D^{2}\right) & \frac{1}{s_{1}} \dot{S}^{1}+\frac{1}{s_{2}} \dot{S}^{2} \\
-\left(s_{1} M^{1}+s_{2} M^{2}\right) & D^{T 1}+D^{T 2}
\end{array}\right)\left(\begin{array}{c}
\dot{u} \\
q
\end{array}\right)=\left(\begin{array}{c}
\dot{u}^{\text {inc }} \\
s_{1} \frac{\partial u^{\text {inc }}}{\partial n}
\end{array}\right)
$$

modified Müller

$$
\left(\begin{array}{cc}
\frac{s_{1}+s_{2}}{2}-\left(s_{1} D^{1}-s_{2} D^{2}\right) & \dot{S}^{1}-\dot{S}^{2} \\
-\left(M^{1}-M^{2}\right) & \frac{s_{1}+s_{2}}{2 s_{1} s_{2}}+\frac{1}{s_{1}} D^{T 1}-\frac{1}{s_{2}} D^{T 2}
\end{array}\right)\left(\begin{array}{c}
\dot{u} \\
q
\end{array}\right)=\left(\begin{array}{c}
s_{1} \dot{u}^{\text {inc }} \\
\frac{\partial u^{\text {inc }}}{\partial n}
\end{array}\right)
$$

modified Burton-Miller

$$
\left(\begin{array}{cc}
\frac{1}{2 c_{1}}-\left(M^{1}+\frac{1}{c_{1}} D^{1}\right) & \frac{1}{2 s_{1}}+\frac{1}{s_{1}} D^{T 1}+\frac{1}{c_{1} s_{1}} \dot{S}^{1} \\
-\frac{1}{2}-D^{2} & \frac{1}{s_{2}} \dot{S}^{2}
\end{array}\right)\left(\begin{array}{c}
\dot{u} \\
q
\end{array}\right)=\left(\begin{array}{c}
\frac{\partial u^{\mathrm{inc}}}{\partial n}+\frac{1}{c_{1}} \frac{\partial u^{\mathrm{inc}}}{\partial t} \\
0
\end{array}\right)
$$

modified standard

$$
\left(\begin{array}{cc}
\frac{1}{2}-D^{1} & \frac{1}{s_{1}} \dot{S}^{1} \\
\frac{1}{2}+D^{2} & -\frac{1}{s_{2}} \dot{S}^{2}
\end{array}\right)\left(\begin{array}{c}
\dot{u} \\
q
\end{array}\right)=\left(\begin{array}{c}
\dot{u}^{\text {inc }} \\
0
\end{array}\right)
$$

where $M^{\nu}$ is the time integral of $N^{\nu}$.

The PMCHWT, Müller and BM formulations are known not to have real fictitious eigenfrequencies in the frequency domain, while the standard formulation does have real fictitious eigenfrequencies [26, 27]. It is therefore expected that the standard formulation is more prone to instability.

We remark that the time differentiated standard integral equation in the modified standard equation (33) has appeared in the paper by Panagiotopoulos and Manolis [14] in the context of elastodynamics in 3D. Also, the combined use of $D, \dot{S}, M$ and $D^{T}$ in 30 , (31) and $(32)$ has been proposed by Abboud et al. [3] and Banjai et al.[21] in different contexts in 3D. Their choices of unknowns are different from ours. To the best of our knowledge, however, these potentials have not been utilised in forms given in (30), (31) and (32) in transmission problems for the wave equation in $2 \mathrm{D}$. 


\subsection{Numerical experiments}

Setting $s_{1}=1, \rho_{1}=1, s_{2}=0.2$ and $\rho_{2}=0.37$, we solve the time domain BIEs in (26)-(33). The incident wave is the quadratic one in (21) and the number of boundary subdivisions, the number of time steps and the time increments are the same as in 2.5. We use piecewise linear time basis functions for $(u, q)$ in the ordinary formulations and for $(\dot{u}, q)$ in the modified formulations.

Fig, 7 and Fig, 8 show the results of $q$ obtained with the ordinary and modified integral equations respectively. We see that the ordinary formulations give unstable results except for the Müller formulation, whereas all the modified formulations provide stable results. Fig.9 shows the distribution

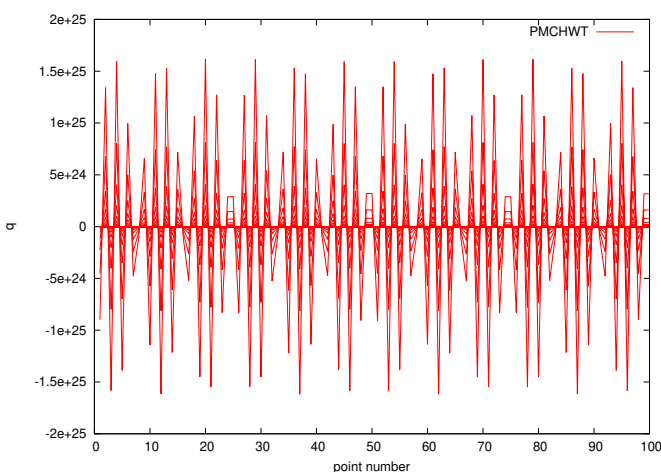

(a) PMCHWT 26

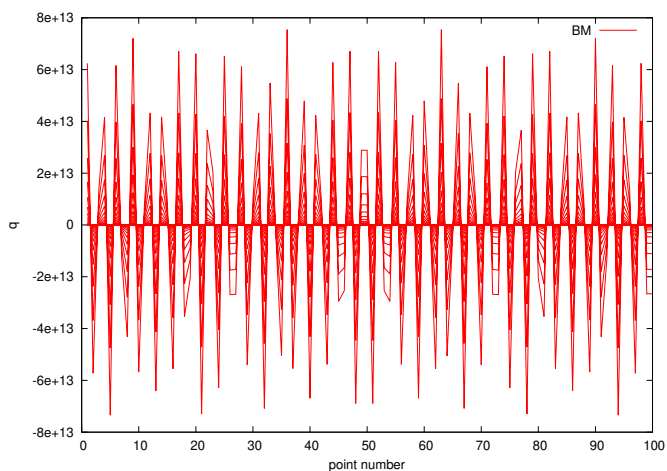

(c) Burton-Miller 28

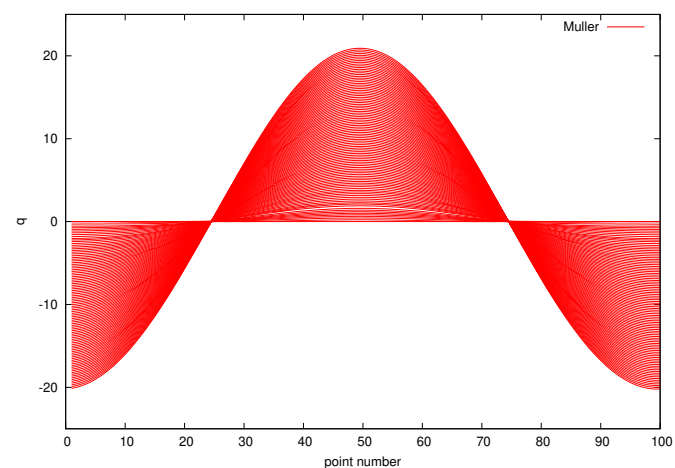

(b) Müller 27)

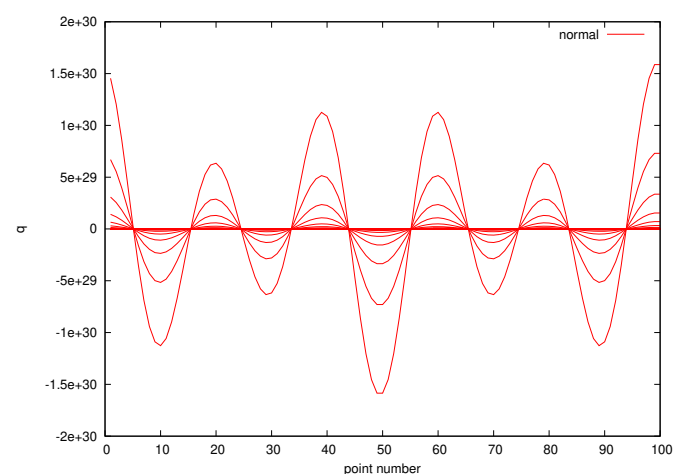

(d) standard BIEs 29

Figure 7: $q$ obtained with ordinary integral equations for transmission problems

of characteristic roots for those formulations which do not include $N$ or $\dot{D}$. 


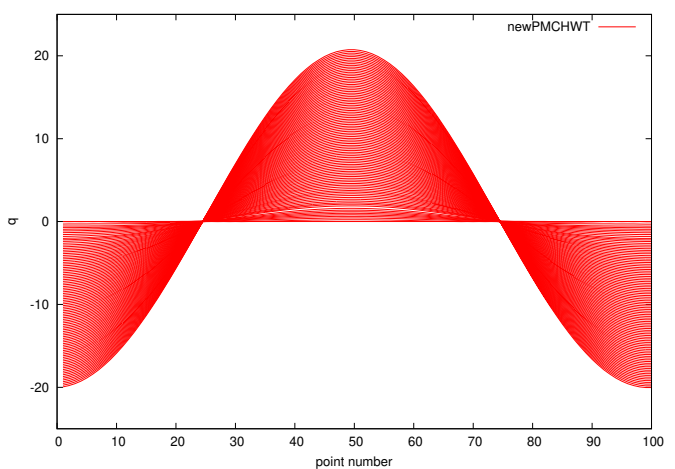

(a) modified PMCHWT (30)

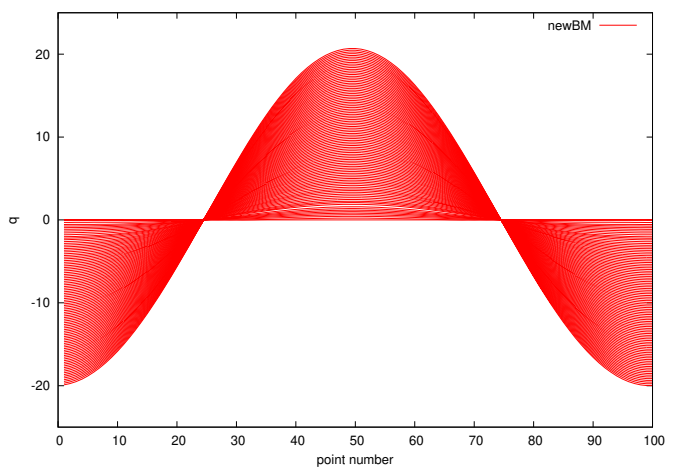

(c) modified Burton-Miller 32

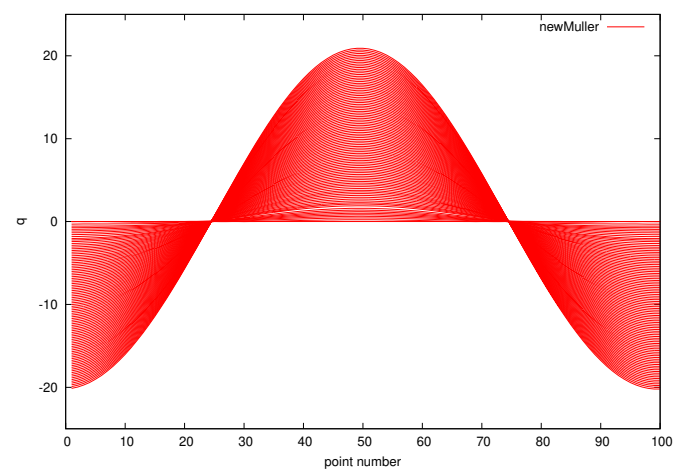

(b) modified Müller (31)

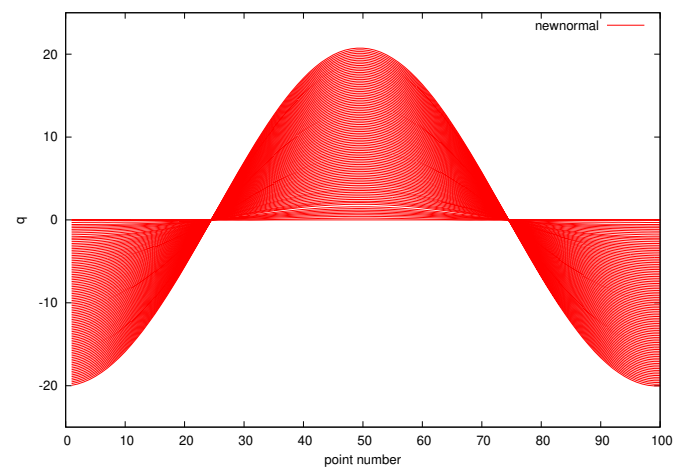

(d) modified standard BIEs (33)

Figure 8: $q$ obtained with modified integral equations for transmission problems

In the modified PMCHWT, for example, they are obtained as the non linear eigenvalues $\left(\Omega\right.$ 's) of the following matrix for one of $n=0, \cdots, n_{\max }$. See (18)- 25):

$$
\begin{aligned}
& \tilde{K}(\Omega ; n)_{\mathrm{PMCHWT}}= \\
& \left.\qquad \begin{array}{cc}
-\left(f_{3}\left(\Omega ; n, c_{1}\right)+f_{2}\left(\Omega ; n, c_{2}\right)\right) & \frac{1}{s_{1}} f_{1}\left(\Omega ; n, c_{1}\right)+\frac{1}{s_{2}} f_{1}\left(\Omega ; n, c_{2}\right) \\
-\left(s_{1} f_{4}\left(\Omega ; n, c_{1}\right)+s_{2} f_{4}\left(\Omega ; n, c_{2}\right)\right) & f_{2}\left(\Omega ; n, c_{1}\right)+f_{3}\left(\Omega ; n, c_{2}\right)
\end{array}\right)
\end{aligned}
$$

The distribution of characteristic roots shown in Fig 9 is seen to be consistent with the time domain results in Figs.7 and 8 . These results also suggest that the use of standard BIEs may not be recommended even after the modification since this formulation has many characteristic roots near the real 


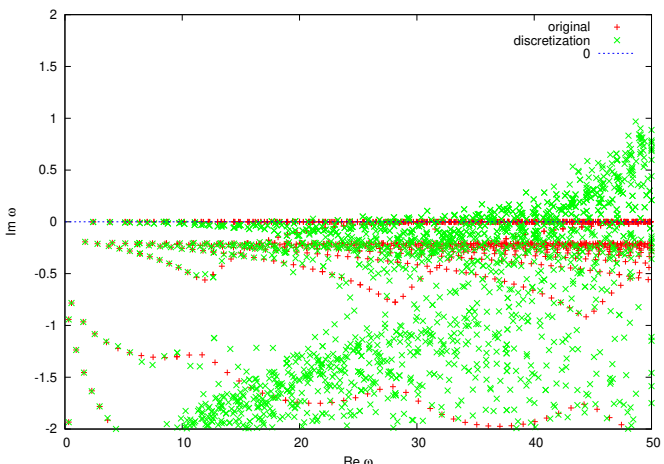

(a) ordinary standard BIEs 29

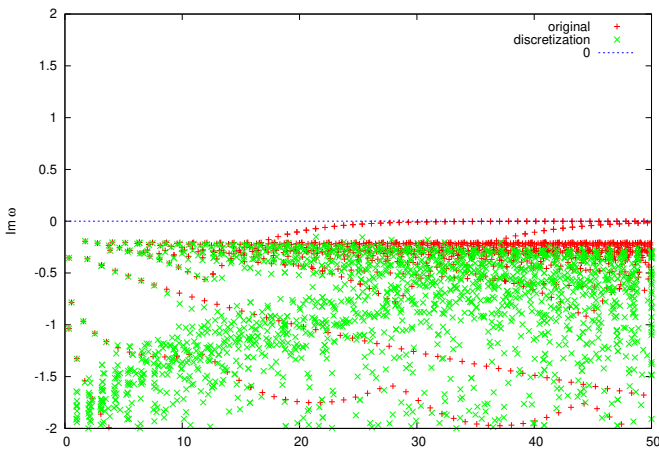

(b) modified PMCHWT 30

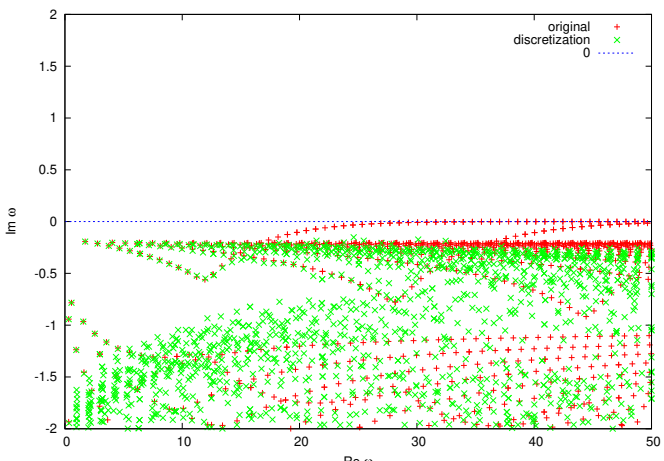

(d) modified Burton-Miller (32)

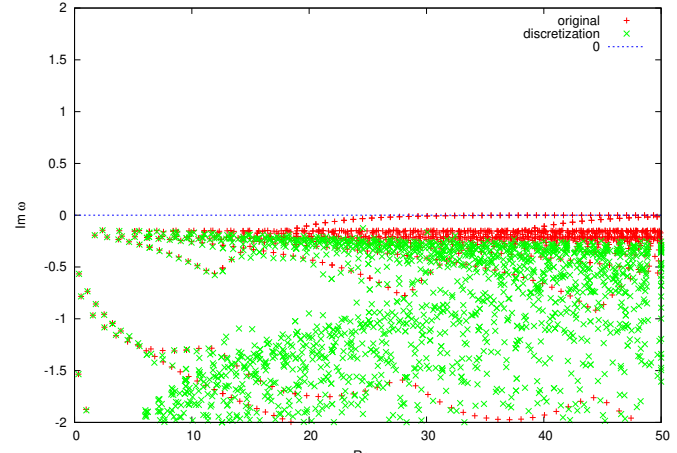

(c) modified Müller (31)

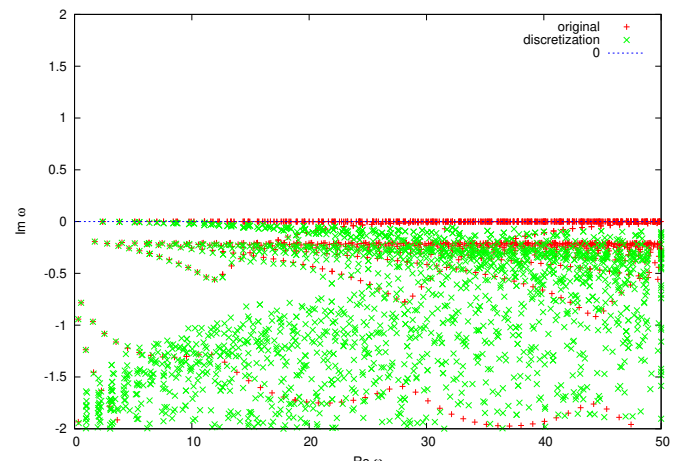

(e) modified standard BIEs 33

Figure 9: Characteristic roots of various integral equations for transmission problems. $\times$ : characteristic roots, + : eigenvalues of frequency domain BIEs 
axis.

Among other three formulations the Müller formulation appears to be better in terms of stability since it gives stable results even without modification, as one sees in Figs.7 and 8. Another reason to prefer Müller is the behaviour of the characteristic equations (34), etc., near $\Omega=0$. As a matter of fact, we can show that $\Omega=0$ is a characteristic root of BM for $n=0$, but other two do not suffer from this problem. However, the characteristic equation (34) for the modified PMCHWT has the following asymptotic behaviour near $\Omega=0$ :

$$
\tilde{K}(\Omega ; n)_{\mathrm{PMCHWT}}=\left(\begin{array}{cc}
o(\Omega) & O(\Omega) \\
O(1 / \Omega) & o(\Omega)
\end{array}\right) \quad n \geq 1
$$

while $\tilde{K}(\Omega ; 0)_{\text {PMCHWT }}=O(1)$. This means that the vector $(0,1)^{T}$ behaves asymptotically like an eigenvector of $(35)$ for $\Omega \approx 0$. This suggests that an arbitrary error of $q$ having a zero spatial mean may persist in the solution of the modified PMCHWT. For the modified Müller equation, however, there is no problem of this kind since we have

$$
\tilde{K}(\Omega ; n)_{\text {Müller }}=\left(\begin{array}{cc}
O(1) & o(1) \\
o(1) & O(1)
\end{array}\right) .
$$

We thus conclude that the modified Müller equation is a better choice than the other two in the cases tested.

To confirm this conclusion, we use the modified PMCHWT and Müller equations to solve the same transmission problem as in Figs.7 and 8 after replacing the incident wave by the quasi-linear one in (22). As has been expected, the modified PMCHWT result includes persistent noise, while the modified Müller result is smooth as shown in Fig.10. Further details of this subject will be presented elsewhere.

\section{Effects of space discretisation}

So far, we have neglected the effect of spatial discretisation in the discussion of stability. This section discusses how the distribution of the eigenvalues is influenced by the space discretisation. We restrict our attention to the circular scatterer case using the Fourier series expansion in order to keep the discussion as analytical as possible so that we can obtain insights. 


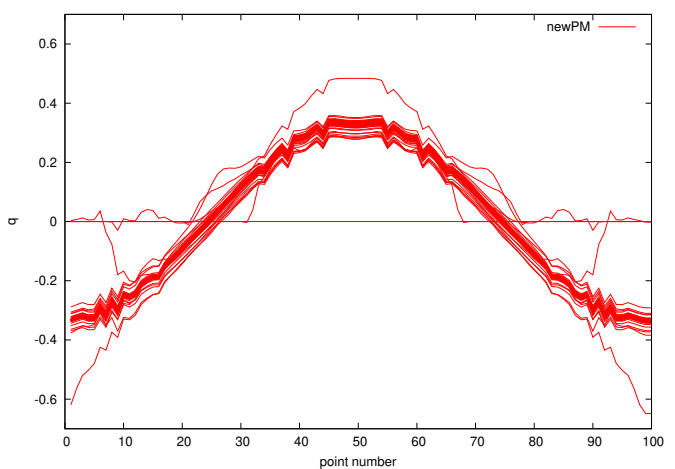

(a) modified PMCHWT

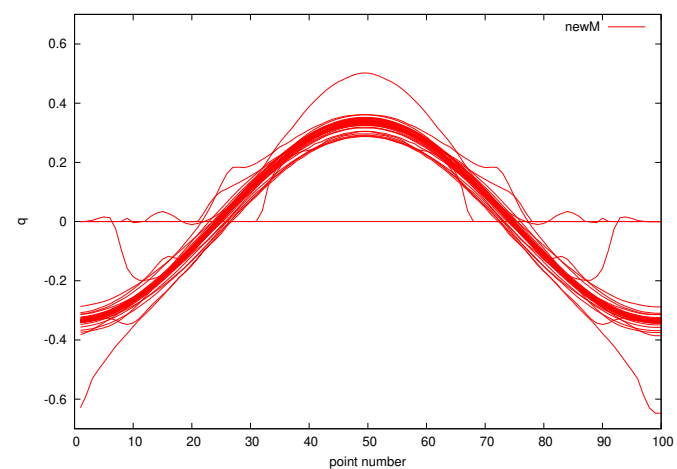

(b) modified Müller

Figure 10: $q$ obtained with modified PMCHWT and Müller formulations

\subsection{Formulation}

We consider a circular boundary having a radius of 1 with $N$ piecewise constant arc elements whose endpoints (angles) are given as $\theta=\theta_{1}, \cdots, \theta_{N}$ $\left(\theta_{N+1}=\theta_{1}\right)$. We consider an integral operator $K(\Omega)$ which maps a function $v$ on the boundary to another function on the boundary. The function $v$ is then approximated with the piecewise constant basis function on each element as follows:

$$
v(\theta) \approx \sum_{p=1}^{N} v_{p} N_{p}(\theta), N_{p}(\theta)=\left\{\begin{array}{cc}
1 & \theta \in\left[\theta_{p}, \theta_{p+1}\right] \\
0 & \text { otherwise }
\end{array}\right.
$$

where $\theta$ is the angular coordinate of the position on the boundary. This is considered to be a reasonable approximation of the discretisation with straight line boundary elements. The basis function is now expanded into the Fourier series given by:

$$
N_{p}(\theta) \approx \sum_{l=-M}^{M} V_{l}^{p} e^{i l \psi}
$$

where $M$ is the truncation number of the infinite Fourier series and $V_{l}^{p}$ is the coefficient of the Fourier series given as follows:

$$
V_{l}^{p}=\frac{1}{2 \pi} \int_{0}^{2 \pi} e^{-i l \psi} N_{p} d \psi=\frac{1}{2 \pi} \int_{\theta_{p}}^{\theta_{p+1}} e^{-i l \psi} d \psi .
$$


Suppose that $K$ is an integral operator for layer potentials such as $\hat{S}, \hat{M}$, etc., in frequency domain. Then the value of $K v$ at a collocation point $\Theta_{n}=\left(\theta_{n}+\theta_{n+1}\right) / 2$ is given as

$$
\left.K v\right|_{\Theta_{n}, n=1, \cdots, N}=\sum_{p=1}^{N} \sum_{l=-M}^{M} e^{i l \Theta_{n}}\{H J\}_{l} V_{l}^{p} v_{p} .
$$

where $\{H J\}_{l}$ stands for the product of Hankel and Bessel functions (or their derivatives) with appropriate coefficients. The $\{H J\}$ 's for the integral operators used in this paper are given as follows:

$$
\begin{aligned}
& S^{\nu} \leftrightarrow H_{n}^{(1)}\left(k_{\nu}\right) J_{n}\left(k_{\nu}\right) \\
& \dot{S}^{\nu} \leftrightarrow-i \Omega H_{n}^{(1)}\left(k_{\nu}\right) J_{n}\left(k_{\nu}\right) \\
& D^{\nu-}, D^{T \nu+} \leftrightarrow k_{\nu} H_{n}^{(1)^{\prime}}\left(k_{\nu}\right) J_{n}\left(k_{\nu}\right) \\
& D^{\nu+}, D^{T \nu-} \leftrightarrow k_{\nu} H_{n}^{(1)}\left(k_{\nu}\right) J_{n}^{\prime}\left(k_{\nu}\right) \\
& M^{\nu} \leftrightarrow-k_{\nu}^{2} H_{n}^{(1)^{\prime}}\left(k_{\nu}\right) J_{n}^{\prime}\left(k_{\nu}\right) / i / \Omega
\end{aligned}
$$

The question of stability of the discretised integral operators in time domain is now reduced to the nonlinear eigenvalue problem for $\Omega$ for the following matrix:

$$
\mathrm{U}\left(\begin{array}{ccc}
\mathcal{D}_{-M} & & 0 \\
& \ddots & \\
0 & & \mathcal{D}_{M}
\end{array}\right) \mathrm{V}
$$

where $\mathrm{V}(\mathrm{U})$ is a $(2 M+1) \times N(N \times(2 M+1))$ matrix whose $(l, p)((n, l))$ components are given by

$$
\mathrm{V}_{l p}=V_{l}^{p} \quad\left(\mathrm{U}_{n l}=e^{i l \Theta_{n}}\right)
$$

and

$$
\mathcal{D}_{l}=\sum_{m=-\infty}^{\infty}\{H J\}_{l}\left(\Omega-\frac{2 m \pi}{\Delta t}\right) \hat{\phi}_{\Delta t}\left(\Omega-\frac{2 m \pi}{\Delta t}\right)
$$

The corresponding matrix for the boundary integral equations for transmission problems can be obtained similarly. We can now solve the non-linear eigenvalue problem given by

$$
K(\Omega) v=0
$$

with the standard SSM. These eigenvalues are called characteristic roots to be consistent with previous sections. 


\subsection{Numerical experiments}

We now show results of some numerical experiments. We consider various integral operators on the unit circle, setting $\rho=1, s=1$ and $\Delta t=2 \pi / 100$, respectively. The mesh on the boundary is uniform with $\theta_{p}=2 \pi p / N, p=$ $1,2, \cdots, N+1$. The number of boundary subdivision $N$ is set to be either $N=100$ or $N=200$. Accordingly, the truncation number of the Fourier series $M$ is set to be $M=10 N+N / 2$. Also, the infinite series $\sum_{m}\{H J\}_{m}$ in (37) and similar ones for transmission problems are truncated with 100 terms since results obtained with 1000 terms were almost identical. We note that it is not very easy to calculate Bessel and Hankel functions of high order with large arguments included in this calculation. This problem is handled with the help of Exflib, a well-known multiple-precision library [31].

Figs.11 show the characteristic roots of various discretised integral operators considered in section 3. The results with $N=100(N=200)$ are shown in triangular (circular) symbols and those without space discretisation (i.e., the characteristic roots given in previous sections, which we call "no space discretisation" in the rest of this paper) are given in cross symbols. It is seen that the property of distributions of characteristic roots does not change very much regardless of the space discretisations. Namely, stable potentials seem to remain stable for reasonable spatial divisions. We also see that the $N=200$ results are closer to the no space discretisation results than those obtained with $N=100$. These observations justify the use of the no space discretisation method in the discussion of the stability of the time domain BEMs.

We next consider transmission problems. Figs. 12 show the characteristic roots of the modified boundary integral equations for transmission problems, i.e., modified PMCHWT (30), Müller (31), BM (32) and standard equations (33). We set $\rho_{1}=1, \rho_{2}=0.37, s_{1}=1, s_{2}=0.2$, and $\Delta t=2 \pi / 100$, respectively as in 4.3 . Once again, the number of the spatial subdivision $N$ does not seem to change the distribution of the characteristic roots qualitatively. We therefore conclude that the stability of these formulations can be inferred from the no space discretisation results. Also, the finer the spatial discretisation the better approximation the no discretisation results become. We thus expect that further spatial discretisation is not likely to affect the stability of the modified boundary integral equations. 


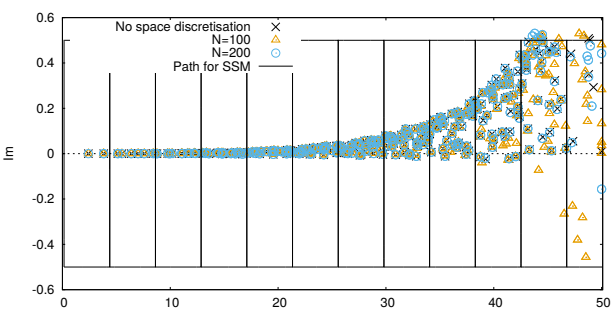

(a) $\stackrel{\text { Ro }}{S}$

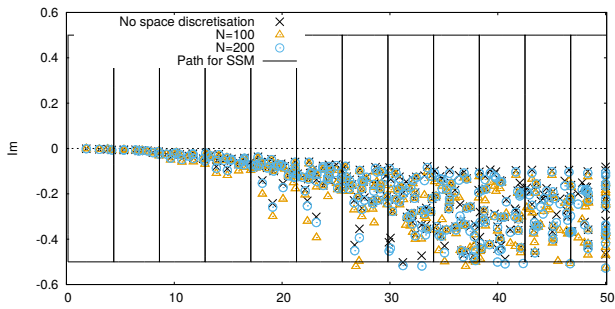

(c) $D^{+}, D^{\text {Re }} D^{T-}$

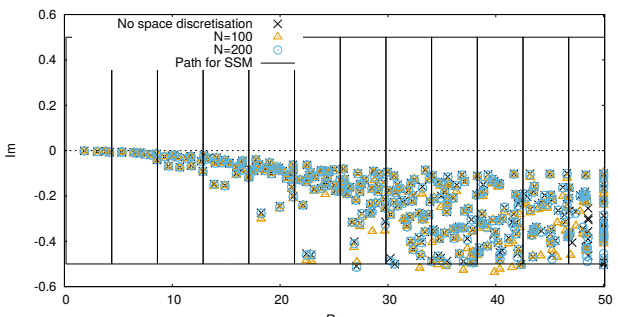

(e) $\stackrel{\text { Re }}{M}$

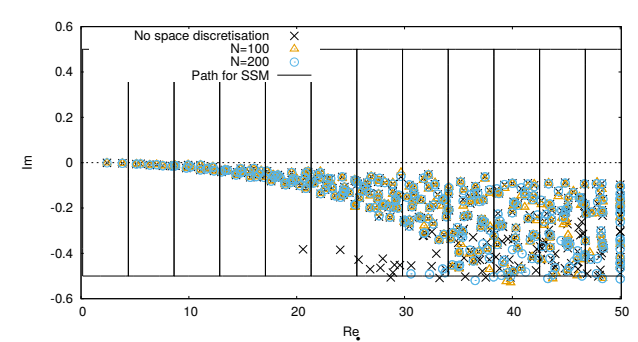

(b) $\stackrel{\text { Re }}{S}$

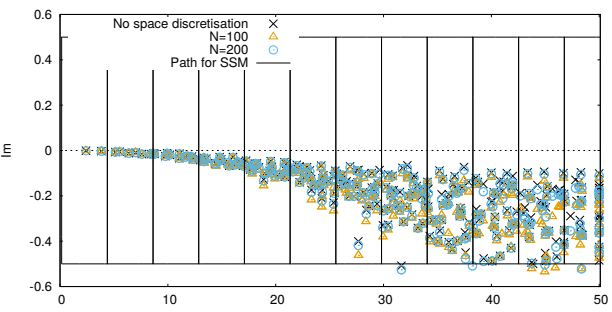

(d) $D^{-}, D^{\text {Re }} D^{T+}$

Figure 11: Characteristic roots of integral operators. $\times$ : no space discretisation, $\triangle$ : $N=100, \circ: N=200$

\section{Non circular boundary}

Finally, we test if the modified formulations remain stable for boundaries other than circle. We consider transmission problems for a "star" (Fig 13(a) given by

$$
\left(x_{1}, x_{2}\right)=((1+0.3 \cos 5 \theta) \cos \theta / 1.3,(1+0.3 \cos 5 \theta) \sin \theta / 1.3)
$$

and a "kite" (Fig,13(b) given by

$$
\left(x_{1}, x_{2}\right)=(0.18(\cos \theta+2(\cos 2 \theta-1)), 0.72 \sin \theta) \text {. }
$$




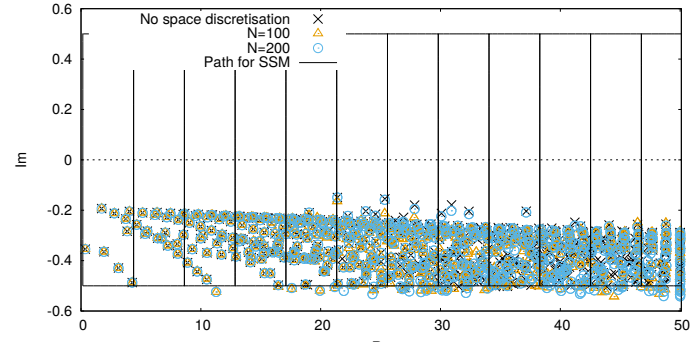

(a) modified $\stackrel{\text { Re }}{\mathrm{P}} \mathrm{MCHWT}$

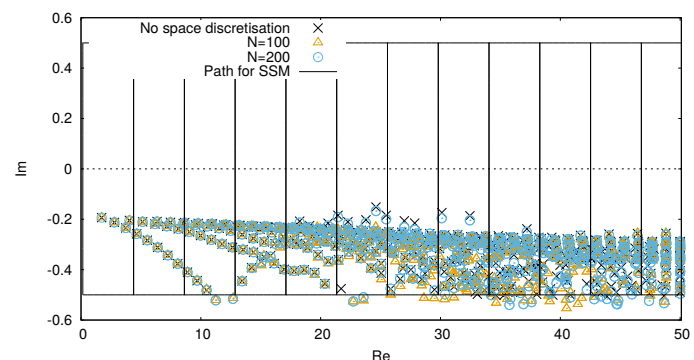

(c) modified Burton-Miller

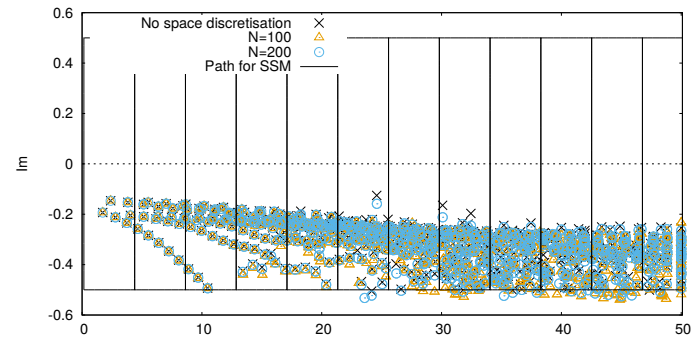

(b) modified Müller

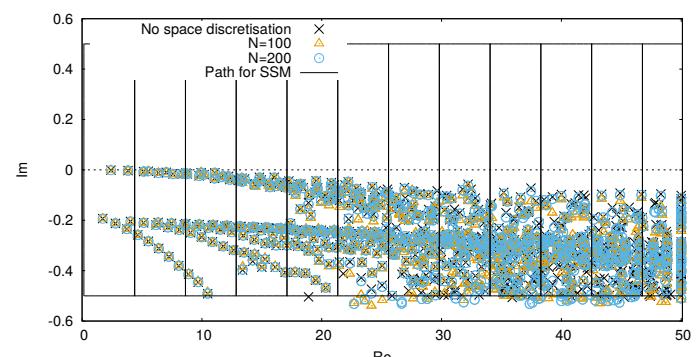

(d) modified standard

Figure 12: Characteristic roots of the modified integral equations for transmission problems. $\times$ : no space discretisation, $\triangle: N=100, \circ: N=200$

The incident wave is the quadratic one in (21) and parameters such as material constants, number of boundary subdivision, $\Delta t$ etc. are the same as those in the transmission problems considered in section 4 . The boundary subdivision is uniform with respect to $\theta$.

Fig,14 and Fig,15 show the distribution of $q$ on the boundary obtained with various formulations. Ordinary formulations except for Müller turn out to be unstable while modified formulations appear to be stable. Also, the results obtained with modified formulations basically agree with each other except for details. These comments apply as well to other cases which are not shown in the paper.

\section{Concluding remarks}

This paper revisited stability issues for BEMs for the two dimensional wave equation in time domain. We presented a stability analysis based on integral equations in frequency domain and showed its validity and usefulness in simple exterior or transmission problems for circular domains. The 


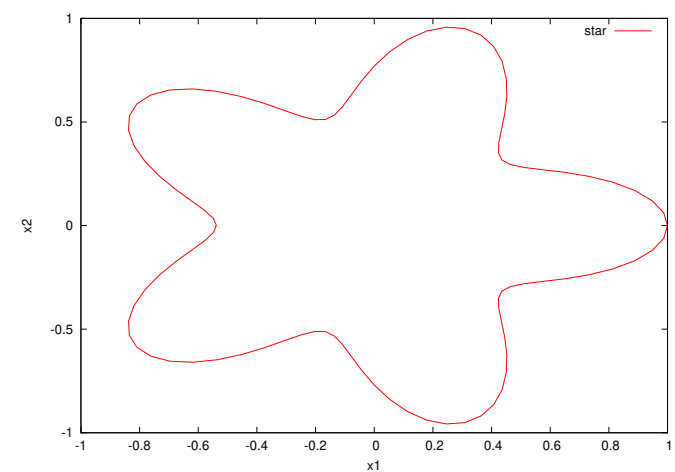

(a) star

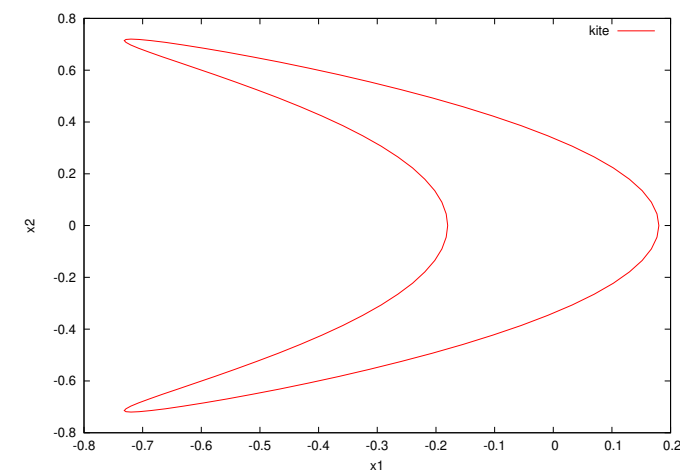

(b) kite

Figure 13: Domains

resulting non-linear eigenvalue problems for the characteristic roots have been solved numerically with SSM. We identified layer potentials which lead to stable integral equations with linear time interpolation for our particular choices of parameters. Combining these potentials, we could formulate stable integral equations for transmission problems which include the velocity and the normal flux of the solution on the boundary as unknowns. Among integral equations considered the Müller formulation was concluded to be a better choice in cases tested. All these modified formulations with similar discretisation conditions were shown to remain stable in transmission problems for star and kite shaped boundaries.

We remark that we have no intention to claim that the combination of particular potentials always leads to stability or that the Müller formulation is always the best choice in transmission problems. What we showed in this paper is the fact that the proposed method of stability analysis in frequency domain is useful in investigating the stability and the accuracy of a time domain BEM for the wave equation in $2 \mathrm{D}$, given particular integral equations and discretisation methods. To be consistent with the purpose of this paper, we have restricted our attention to simple problems where we can utilise analytical tools as much as possible. Also, the numerical examples presented have been limited to small number of cases.

As future directions, we can mention the following:

1. Apply the stability analysis in frequency domain to problems with non-circular boundaries and carry out more extensive numerical ex- 


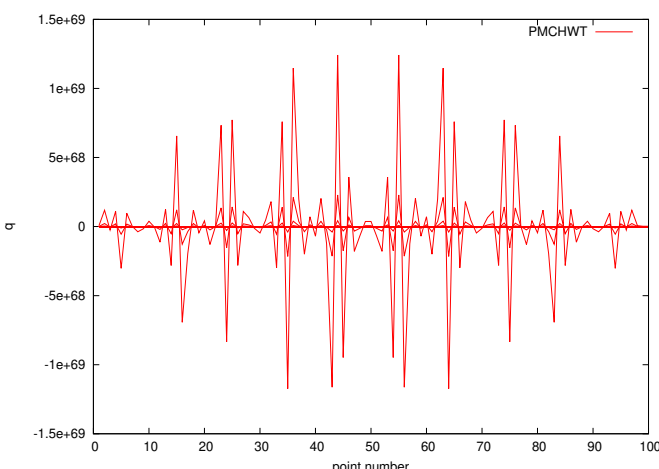

(a) PMCHWT

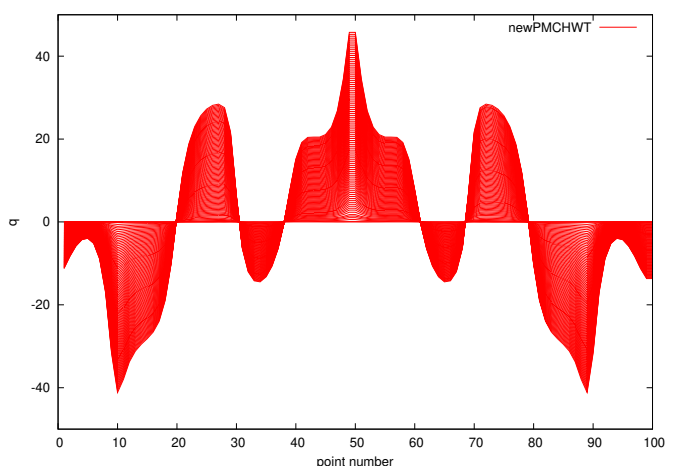

(c) modified PMCHWT

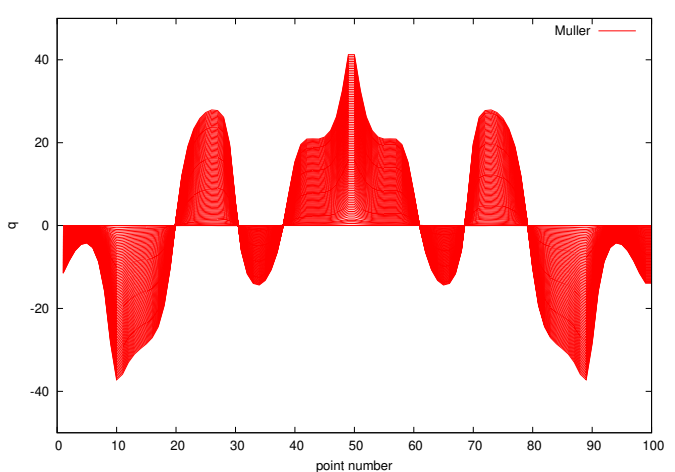

(b) Müller

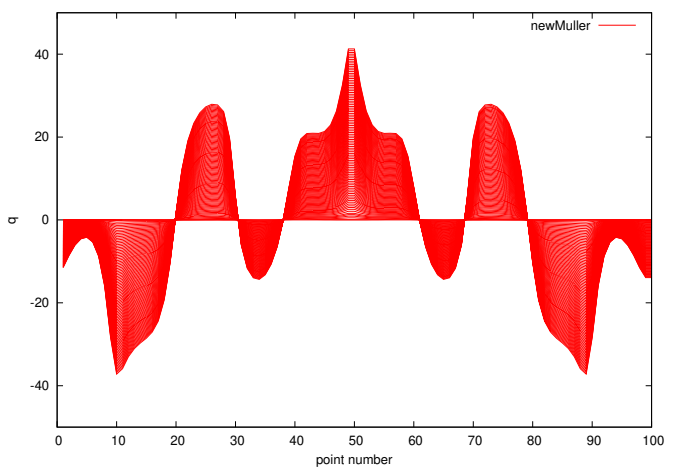

(d) modified Müller

Figure 14: Transmission problems for "star"

periments.

435

440

2. Investigate the stability of modified time domain formulations for exterior and transmission problems in 3D.

3. Consider the stability of interior problems in which true eigenvalues may cause instability[10].

4. Study the robustness of the algorithms for the time domain integral equations.

The first item will include numerical treatment of $(13)$ in which one considers integral equations having the function in (15) as the kernel instead of the fundamental solution in BIEs on general boundaries. As regards the 2nd item, we have already started investigations which so far seem to tell that conclusions similar to those in this paper hold in 3D as well. Notice, however, 


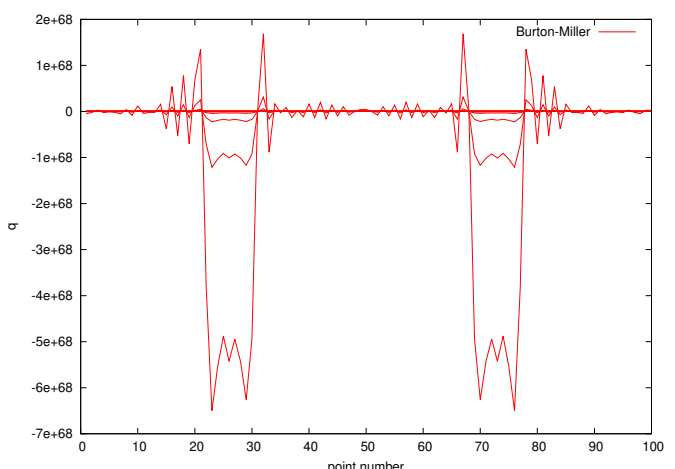

(a) BM

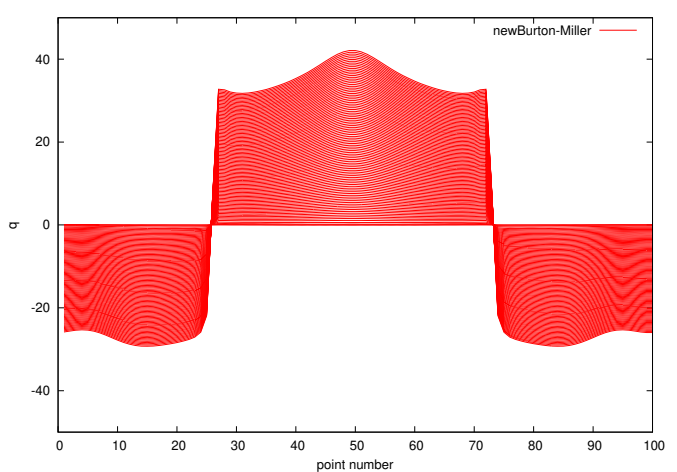

(c) modified BM

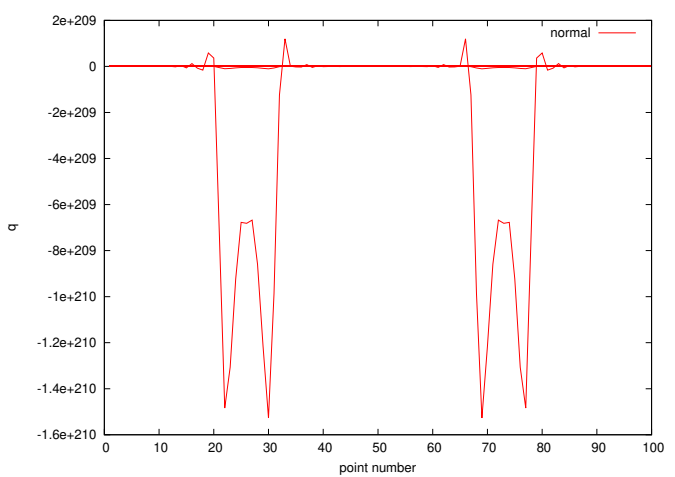

(b) standard

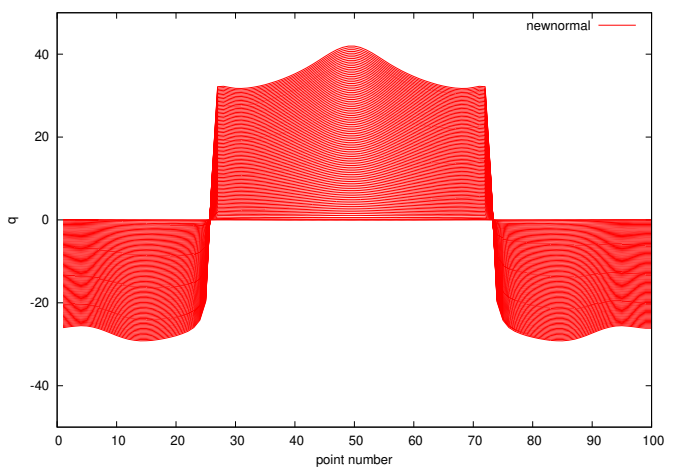

(d) modified standard

Figure 15: Transmission problems for "kite"

that the use of the time domain stability analysis based on linear eigenvalue problems and (11) may be simpler than the frequency domain approach in 3D (and, indeed, have already been utilised by many authors including Walker et al.[24], etc., as have been mentioned) because of the finite "tail" of the fundamental solution. We remark, however, that the direct application of SSM to 12 and the use of proposed frequency-domain stability analysis in (13) in 3D are still worth trying. Concerning the 4th item, we have carefully avoided this issue in the present investigation. In real world applications, however, the robustness of the algorithm is very important since one may have to use numerical integrations, truncated time steps, fast methods etc., which will inevitably introduce errors. 


\section{Acknowledgement}

This work has been supported by JSPS KAKENHI Grant Number $18 H 03251$.

\section{Appendix A. Further numerical studies of (23)}

Although the purpose of this paper is not to recommend particular time domain integral equations, it might be of interest to carry out further numerical experiments on the behaviour of (23) which may not be standard but appears to be promising among integral equations tested in 2.5. We consider the same exterior Dirichlet problem for a unit circle as has been considered in 2.5 with the same material constants, initial condition and boundary condition. The incident wave is the quasi-linear one given in (22). The boundary is discretised with piecewise constant boundary elements. The time basis function is piecewise linear and the time increment is $\Delta t=\pi / 50$ unless stated otherwise. The coupling constant $\alpha$ in (23) is 1 .

We first remark that the conditioning of the matrix equations to be solved in ordinary time domain BEMs for the wave equation is not a big concern since these equations are usually sparse and can easily be inverted with direct solvers. We have checked if this remains true in (23) which is a combined equation. To this end, we have computed the condition numbers (with respect to 1 norm) of the discretised equations corresponding to (5), (6), (7), (8) and (23). The obtained condition numbers of these equations are 1.2629, $1.9821,1.0087,1.3227$ and 1.3217 , respectively, showing that the combined integral equation in (23) gives an equally well-conditioned algebraic equation as in other standard integral equations.

We next examine the effect of refined spatial meshes keeping the time increment unchanged. In these cases the distribution of the characteristic roots shown in Fig. 5(b) remains applicable, indicating that the stability of the numerical schemes is expected. To check this conclusion, we solved (23) numerically with spatial divisions increased to $N=200,300$ and 400. All these computations gave stable results as have been expected. We here show the result for $N=400$ in Fig. A.16. To check the accuracy, we have computed the error $\epsilon(t)$ defined by

$$
\epsilon(t)=\frac{\left\|q(t)-q_{\mathrm{exact}}(t)\right\|}{\left\|q_{\mathrm{exact}}(t)\right\|}
$$

where $\|\cdot\|$ stands for the $L^{2}$ norm on the boundary and $t$ is taken larger than the arrival time of the incident wave. The result is shown in Fig. A.17(a). 


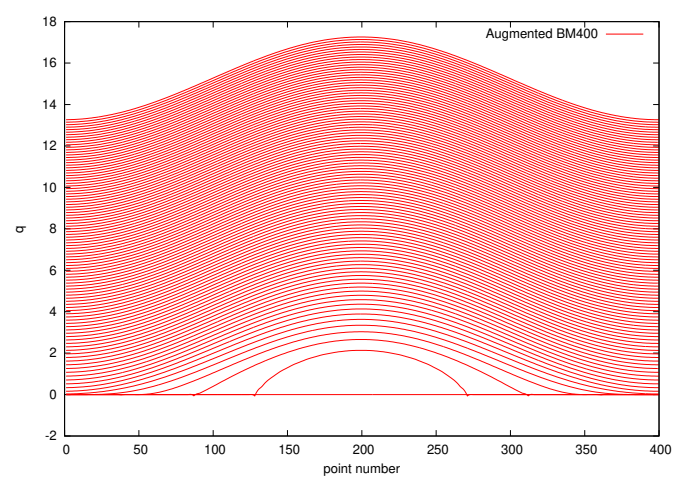

Figure A.16: $q$ obtained with eq. 23 with $N=400$

The curves marked $\mathrm{Nx} N$ stand for results with given number of boundary

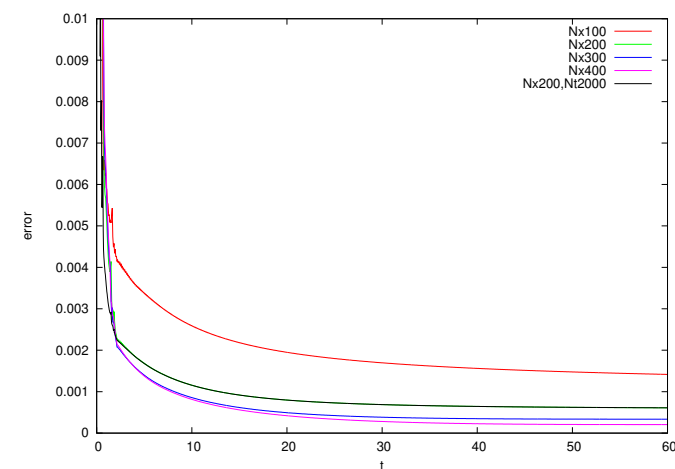

(a) Error

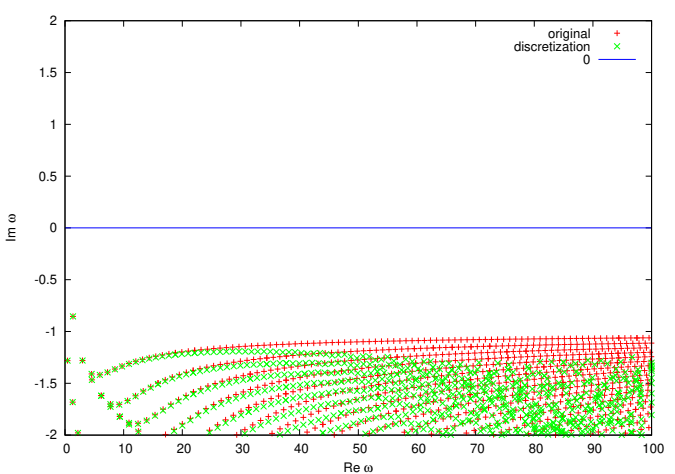

(b) Characteristic roots

Figure A.17: (a)Error $\epsilon(t)$ of the solution of eq. 23 obtained with $N=100,200,300$ and 400, (b)Characteristic roots $(\times)$ and eigenvalues of the frequency domain BIE $(+)$ for $\Delta t=\pi / 100$

elements $N$. For $N=200$ we have also solved (23) with $\Delta t=\pi / 100$ (but with the incident wave unchanged) for 2000 time steps, and the result is marked as $N x 200, N t 2000$ in Fig. A.17(a), Fig. A.17(b) shows the corresponding distribution of characteristic roots for $\Delta t=\pi / 100$.

Fig. A $17(\mathrm{a})$ shows that the error decreases steadily as $t$ or $N$ increases. Also, the accuracy of solutions seems to depend on $\Delta t$ only when $t$ is small with $N=200$. The result in Fig. A.17(b) is consistent with the stability of 
the time domain computation with $\Delta t=\pi / 100$ and $N=200$.

We remark that the effect of the value of $\alpha$ on the accuracy, stability etc. of the solutions has not been examined so far. Selecting an optimum $\alpha$ remains as a future subject.

\section{References}

[1] Bamberger A, Ha Duong T. Formulation variationnelle espace-temps pour le calcul par potentiel retardé de la diffraction d'un onde acoustique (I). Math Meth Appl Sci 1986;8:405-35.

[2] Aimi A, Diligenti M, Guardasoni C. On the energetic Galerkin boundary element method applied to interior wave propagation problems. J Comp Appl Math 2011;235:1746-54.

[3] Abboud T, Joly P, Rodríguez J, Terrasse I. Coupling discontinuous Galerkin methods and retarded potentials for transient wave propagation on unbounded domains, J Comp Phys 2011;230:5877-907.

[4] van 't Wout E, van der Huel DR, vander Ven H, Vuik C. Stability analysis of the marching-on-in time boundary element method for electromagnetics. J Comp Appl Math 2016;294:358-71.

[5] Davies PJ, Duncan DB. Stability and convergence of collocation schemes for retarded potential integral equations. SIAM J Num Anal 2004;42:1167-88.

[6] Costabel M, Sayas F-J. Time-dependent problems with the boundary integral equation method. Chapter 24, Encyclopedia of Computational Mechanics, 2nd Ed., Vol.2, Part 2, Eds. Stein E., de Borst R., Hughes TJR. Chichester: Wiley; 2018.

[7] Parot JM, Thirard C, Puillet C. Elimination of a non-oscillatory instability in a retarded potential integral equation. Eng Anal Boundary Elements 2007;31:133-51.

[8] Parot JM, Thirard C. A numerical algorithm to damp instabilities of a retarded potential integral equation. Eng Anal Boundary Elements 2011;35:691-9. 
[9] Jang H-W, Ih J-G. Stabilization of time domain acoustic boundary element method for the exterior problem avoiding the nonuniqueness. J Acoust Soc Am 2013;133:1237-44.

[10] Jang H-W, Ih J-G. Stabilization of time domain acoustic boundary element method for the interior problem with impedance boundary conditions. J Acoust Soc Am 2012;131:2742-52.

[11] Pak RYS, Bai X. A regularized boundary element formulation with weighted-collocation and higher-order projection for 3D time-domain elastodynamics. Eng Anal Boundary Elements 2018;93:135-42.

[12] Bai X, Pak RYS. On the stability of direct time-domain boundary element methods for elastodynamics. Eng Anal Boundary Elements 2018;96:138-49.

[13] Mansur WJ, Carrer JA, Siqueira EFN. Time discontinuous linear traction approximation in time-domain BEM scalar wave propagation analysis. Int J Num Meth Eng 1998;42:667-83.

[14] Panagiotopoulos C, Manolis GD. Three-dimensional BEM for transient elastodynamics based on the velocity reciprocal theorem. Eng Anal Boundary Elements 2011;35:507-16.

[15] Ergin AA, Shanker B, Michielssen E. Analysis of transient wave scattering from rigid bodies using a Burton-Miller approach. J Acoust Soc Am 1999;106:2396-404.

[16] Chappell DJ, Harris PJ, Henwood H, Chakrabarti R. A stable boundary element method for modeling transient acoustic radiation. J Acoust Soc Am 2006;120:74-80.

[17] Chappell DJ, Harris PJ. On the choice of coupling parameter in the time domain Burton-Miller formulation. Q J Mech Appl Math 2009;624:43150 .

[18] Zhang Y, Bi C-X, Zhang Y-B, Zhang X-Z. Horn effect prediction on the time domain boundary element method. Eng Anal Boundary Elements 2017;82:79-84. 
[19] Lubich C. On the multistep time discretization of linear initial- boundary value problems and their boundary integral equations. Num Math 1994;67:365-89.

[20] Sayas F-J. Retarded potentials and time domain boundary integral equations. Switzerland: Springer; 2010.

[21] Banjai L, Lubich C, Sayas F-J. Stable numerical coupling of exterior and interior problems for the wave equation. Numer Math 2015;129:611-46.

[22] Schanz M. Wave propagation in viscoelastic and poroelastic continua: a boundary element approach, Lecture Notes in Applied Mechanics. Berlin, Heidelberg, New York: Springer; 2001.

[23] Wang H, Henwood DJ, Harris PJ, Chakrabarti R. Concerning the cause of instability in time-stepping boundary element methods applied to the exterior acoustic problem. J Sound Vibration 2007;305:289-97.

[24] Walker SP, Bluck MJ, Chatzis I. The stability of integral equation time-domain computations for three-dimensional scattering; similarities and differences between electrodynamic and elastodynamic computations. Int J Num Modelling, Electronic Networks, Devices and Fields 2002;15:459-74.

[25] Asakura J, Sakurai T, Tadano H, Ikegami T, and Kimura K. A numerical method for nonlinear eigenvalue problems using contour integrals. JSIAM Letters 2009;1:52-5.

[26] Misawa R, Niino K, Nishimura N. An FMM for waveguide problems of 2-D Helmholtz' equation and its application to eigenvalue problems. Wave Motion 2016;63:1-17.

[27] Misawa R, Niino K, Nishimura N. Boundary integral equations for calculating complex eigenvalues of transmission problems. SIAM J Appl Math 2017;77:770-88.

[28] Engquist B, Majda A. Absorbing boundary conditions for the numerical simulation of waves. Math Comp 1977;31:629-51.

[29] Martin PA. Multiple scattering: interaction of time-harmonic waves with $N$ obstacles. Cambridge: Cambridge University Press; 2006. 
[30] Epstein CL, Greengard L Hagstrom T. On the stability of time-domain integral equations for acoustic wave propagation. Discrete Cont Dyn Sys-A 2016;36:4367-382.

[31] http://www-an.acs.i.kyoto-u.ac.jp/ fujiwara/exflib/ 